\title{
Advanced Characterisation Techniques for Nanostructures
}

\author{
Brian Freeland, Inam Ul Ahad, Greg Foley \& Dermot Brabazon
}

3.1 Measurement of the topology of nanostructures

3.1.1 Field Emission Scanning Electron Microscope (FESEM)

3.1.2 Scanning probe microscopy (SPM)

3.1.3 Scanning Tunnelling Microscope (STM)

3.1.4 Atomic Force Microscope (AFM)

3.1.5 Optical microscopes

3.1.5.1 Confocal microscopy

3.1.5.2 Near Field Scanning Optical Microscopy (NSOM)

3.1.6 Dynamic light scattering (DLS)

3.2. Measurement of nanostructures internal geometries

3.2.1 Transmission Electron Microscope (TEM)

3.2.2 Focused Ion Beam (FIB)

3.2.3 X-ray diffraction (XRD)

3.2.4 Mercury Porosimetry

3.3 Measurement of composition of nanostructures

3.3.1 Energy dispersive X-ray spectroscopy (EDS)

3.3.2 X-ray photoelectron spectroscopy (XPS)

3.3.3 Secondary Ion Mass spectroscopy (SIMS)

3.3.4 Auger Electron spectroscopy (AES)

\subsection{Conclusion}




\begin{abstract}
This chapter presents some of the most important currently utilised techniques for the characterisation of nanostructures and nanoparticles. The techniques presented here are grouped into categories of topology, internal structure and compositional investigation. Topological techniques presented here include field emission scanning electron microscopy (FESEM), scanning probe microscopy (SPM), and optical microscopy (confocal and NSOM), and particle size distribution with dynamic light scattering (DLS). Internal structure techniques presented include transmission electron microscope (TEM), magnetic resonance force microscope (MRFM) and X-ray diffraction (XRD). Compositional techniques presented include X-ray photoelectron spectroscopy (XPS), energy dispersive x-ray spectroscopy (EDS), secondary ion mass spectroscopy (SIMS) and Auger electron spectroscopy (AES). To highlight the current capabilities and applications of these techniques, case studies from recent literature are presented.
\end{abstract}

\title{
3.1 Measurement of the topology of nanostructures
}

\subsubsection{Scanning Electron Microscope (SEM)}

Scanning electron microscopes (SEM) have been used by researchers since 1935 to examine micrometer scale structures and is currently the most widely used non-optical microscopy technique. In the past fifteen years, more focus has been brought on its application towards nanostructures. This technique allows for a comparably large sample window and for dimensional measurements to be performed. The principle of operation of the SEM involves a focused electron beam which is fired over the surface of the sample, resulting in electrons being emitted from the surface and are then collected by a positively charged detector grid. These are termed secondary 
electrons. These secondary electrons are recorded for the scanned surface and provide a surface topology. The factors that affect SEM resolution are the primary electron beam spot size and the volume of material with which the electrons interact. In order to ensure best possible resolutions, a high accelerating voltage should be employed (20-30kV), along with a smaller current and small spot size. Care is needed however in some cases to avoid high electron energies where these can modify or destroy the material structure to be observed. Tungsten and carbon are the traditional emission element filaments used for SEM with $\mathrm{LaB}_{6}$ emission sources being commonly adopted now due to their offering of longer gun lives and to not necessitating nitrogen cooling. Another type of emitted electrons "backscattered electrons" are collected by a backscatter collector, they are high energy electrons resultant of the incident electron beam. The response of these electrons is directly proportional to the density of atoms in the material, allowing surface composition, via relative density difference, to be visualised.

\section{SEM case studies}

Recently the SEM technique has been applied towards a range of applications such as determining the effects of gelling agents on the morphology of $\mathrm{ZnSnO}_{3}$ nanostructures [1], examining the surface morphology and nano surface features of flat objects [2] and measuring the diameters of silicon, latex and gold spherical nanoparticles with mean diameter of $15 \mathrm{~nm}$ [3].

It has been observed that SEM performance can be increased by utilising the secondary electron detector at low magnifications to image $\mathrm{TaS}_{2}$ nano tube bundles as seen in figure 3.1. This configuration allowed for larger scan areas and faster processing times. A $10 \mathrm{kV}$ beam voltage was used with a magnification of $12.4 \mathrm{k} \times$ [4]. 


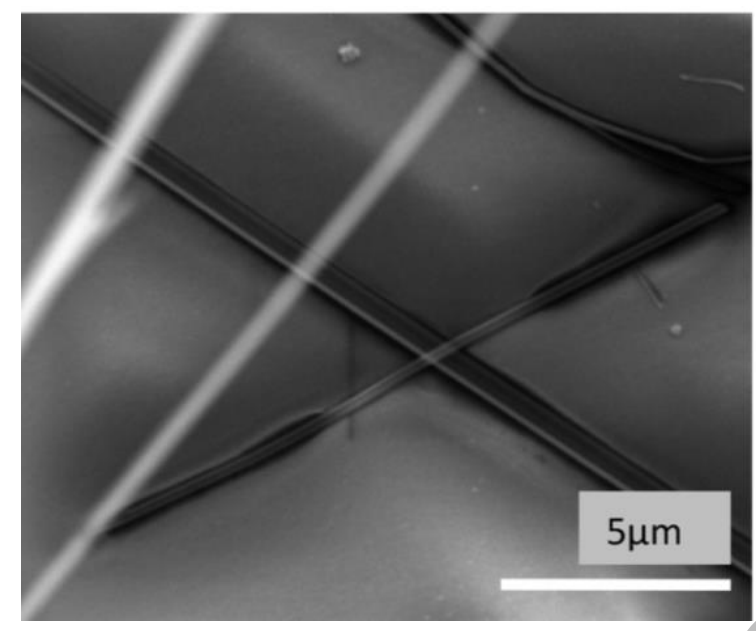

Figure 3.1 SEM image of a silica fragment within a $\mathrm{TaS}_{2}$ nanotube bundle [4].

The SEM typically has at best a spatial resolution of $1-10 \mathrm{~nm}$ and therefore it is generally used to characterise structures greater than $10 \mathrm{~nm}$. Over the years efforts have been made to break this 10nm barrier. Recently Villarrubia et al. (2015) have proposed a technique to improve the resolution of SEM inspection of lithographically patterned $\mathrm{SiO}_{2}$ used by Intel.

The authors fitted the measured intensity vs. position to a physics-based model which included the lithography lines' dimensions as parameters. A 3D model for patterned $\mathrm{SiO}_{2}$ was developed as shown in figure 3.2. They concluded that with the aid of the model, sub-nanometer resolution could be achieved, an improvement of almost ten-fold. 

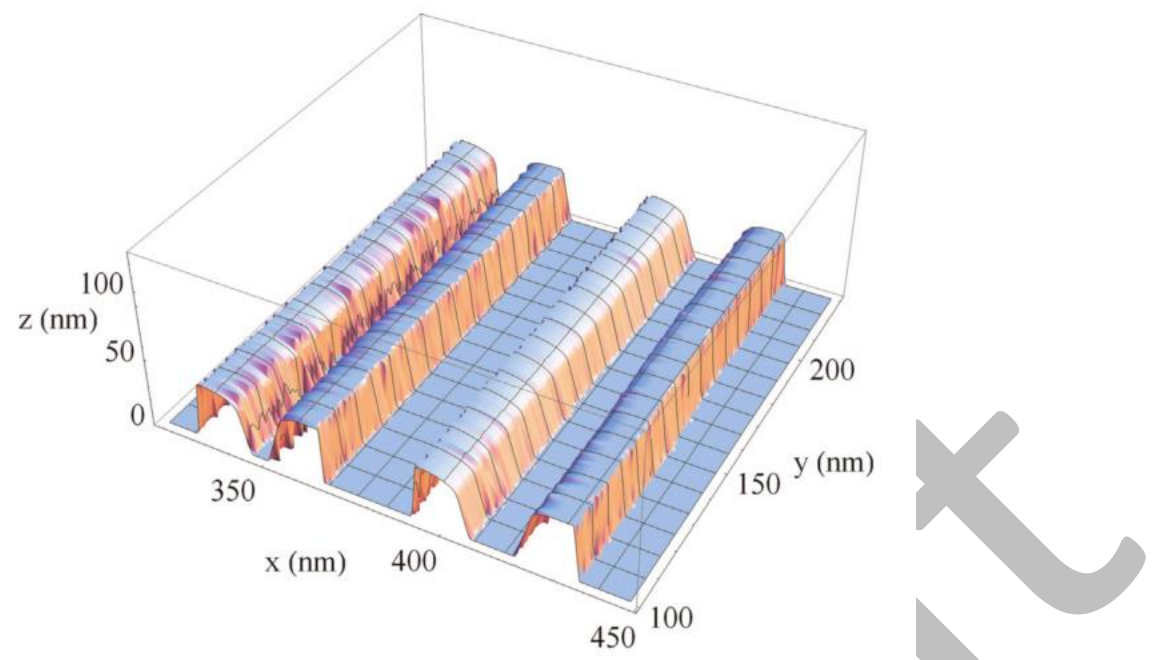

Figure 3.2 SEM generated 3D reconstruction data of lithography produced $\mathrm{SiO}_{2}$ channels [5].

\subsubsection{Field Emission Scanning Electron Microscope (FESEM)}

A field emission cathode in the electron gun of a SEM provides narrower probing beams resulting in both improved spatial resolution and less sample charging. Such systems are designated as Field Emission Scanning Electron Microscopes (FESEM). To achieve this increased electron focusing a different gun design is required [6]. In this design, electrons are expelled by applying a high electric field very close to the filament tip. The size and proximity of the electric field to the electron reservoir in the filament controls the degree to which electrons tunnel out of the reservoir. One type of field emission gun commonly used is known as the Schottky in-lens thermal FESEM electron gun Cold gun alternatives are available for even finer FESEM resolution; however, these suffer rapid degradation and can therefore lead to expensive operation due to relatively frequent placement. The field emission guns have higher stability, can allow higher current and hence provide a smaller spot size. Under good operating conditions a typical FESEM resolution of $1 \mathrm{~nm}$ is achievable. Elements that add to improved operation and FESEM resolution include designs with a beam booster to maintain high beam energy, an electromagnetic multi-hole beam aperture 
changer, a magnetic field lens, and a beam path has been designed to prevent electron beam crossover.

Nanostructures have been characterised by FESEM in different morphological formations including nanoflowers [7], nanosheets [8], nanoparticles [9], and thin films [10]-[13].

Molybdenum disulphide $\left(\mathrm{MoS}_{2}\right)$ nanosheets exhibit interesting conductive/semiconductive, magnetic, photoluminescence, photocatalytic, and field - effect transistor properties. The properties of $\mathrm{MoS}_{2}$ nanosheets depends upon the method used to generate them and depending upon the structural properties, these nanosheets can be used for applications in optoelectronics, energy harvesting, and spinelectronics (fluxtroincs) etc. The characterisation of $\mathrm{MoS}_{2}$ nanosheets morphology has been performed by FESEM in various studies [14]-[18].

\section{FESEM case studies}

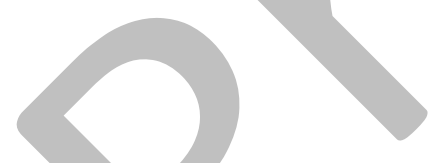

In one study, nanostructured Tin(II) sulphide (SnS) thin film was deposited on FTO glass substrate by electro-deposition (ED)with and with ultrasound assistance electro-deposition (UAED) [19]. Figure 3.4 shows the FESEM micrographs of the ED-SnS and the UAED-SnS produced nanostructures [19]. The effect of using ultrasound waves during electrodeposition on the morphology of the nanostructured SnS films can be clearly seen. The FESEM images showed that without using ultrasound waves, the deposited SnS film had grain-like morphology. Using ultrasound assistance during electrodeposition, the FESEM showed the formation of two distinct nanostructures. The first type was in a formation of $20 \mathrm{~nm}$ to $60 \mathrm{~nm}$ thin plane nanostructures. The second type was in cubical rods type structures with less than one micron height [19].

Other workers have applied FESEM characterisation for the implementation of cryo-SEM natural state colloidal solution capturing and studied the process of simultaneous deposition by immersion plating of palladium and silver seeds from 1 to $100 \mathrm{~nm}$ in size on porous silicon $[5,6]$. 

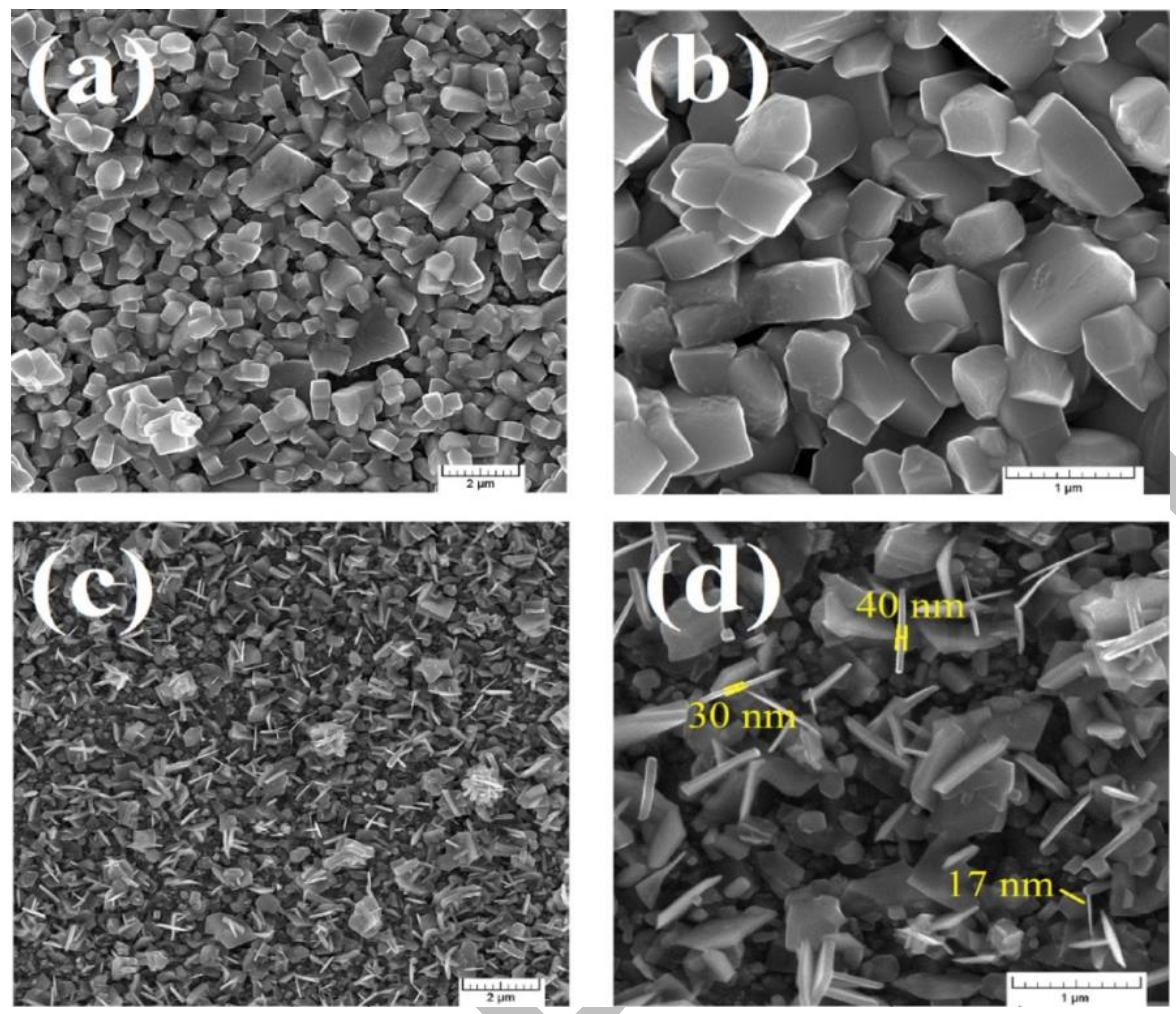

Figure 3.3 FESEM micrographs of the (a), (b) the ED-SnS and

(c), (d) the UAED-SnS produced nanostructures [19].

\subsubsection{Scanning probe microscopy (SPM)}

Scanning probe microscopy is the general term associated with several techniques notably scanning tunnelling microscopy (STM) and atomic force microscope (AFM) discussed in this chapter. Both techniques feature a sharp probe with a point diameter as low as one atom is passed over the sample's surface via piezoelectric actuators in order to visualise the surface features.

\subsubsection{Scanning Tunnelling Microscope (STM)}

Binnig and Rohrer developed the scanning tunnelling microscopy technique back in 1981 and their work lead to a Nobel prize in 1986. The most essential component of an STM is the probe which 
is sharpened to one atom width. Typically, the tip material selection is limited to tungsten, platinum-iridium, gold or carbon Nano-tube. Recently there have been developments towards graphene coated probe tips, reducing costs compared with standard Pt-Ir probes [20].

The tip is scanned over the surface to be characterized via three piezoelectric actuators orientated in three dimensions to control the probes position with respect to the surface. The sample surfaces must be conductive or semi conductive for the technique to operate as a voltage is applied between the tip and the sample, a current is generated via electron tunnelling, this current is monitored, as the distance between the probe tip and the surface changes the resulting current changes in proportion. Therefore the dimensional precision of the probe tip is essential to the precision of the technique. STM can achieve atomic scale visualisation and this accuracy is due to the stages that can provide $0.1 \mathrm{~nm}$ lateral and $0.01 \mathrm{~nm}$ depth resolution. To achieve these resolutions, vibration must be minimised and consequently the systems are mounted on weighted or specialised antivibration plates while springs are used as part of the tip mechanism for vibration isolation.

For the SEM, there are two modes of operation: constant height and the constant current mode. In constant current mode the tip is scanned over the surface and its position in the vertical axis is altered to keep the current constant. This change in tip height is recorded and used to map the surface. With constant height mode, the height of the tip and the voltage is maintained at a constant. The change in current required to keep the voltage constant is related to local charge density to provide a record of the surface profile. Constant height mode provides faster scan times compared with constant current, and is the preferred mode for larger sample windows. 


\section{STM case studies}

Krupski utilised STM to determine the growth of ultra-thin Au films deposited on a Mo (110) substrate at high temperatures (300k) [21]. They found that by using differing Au depositions, they were able to determine the morphology of the Au layers. This allowed for terracing with a width of $25-180 \mathrm{~nm}$ to be characterised on the surface [21]. More recently Huerta et al. (2016) investigated the multilayer growth of 4-aminothiophenol (4-ATP) on an iodine-modified Au (100) substrate. The interaction of ATP molecules and the iodine monolayer was determined oyer time, the STM providing images of the structure formation from single molecules into chains, which could lead to uses in the production of solid phase micro extraction coatings [22].

Figure 3.4 shows the STM images of APT on an iodine doped Au layer. Figure 3.4 (a) taken at a potential of $+0.1 \mathrm{~V}$ with an image size of $130 \times 130 \mathrm{~nm}^{2}$ shows the multilayer growth that occurred, including the formation of holes, pits and trenches between the deposited molecules [23]. The higher resolution image of figure 3.4 (b) with an area of $8.1 \times 8.1 \mathrm{~nm}^{2}$ shows the formation of surface features such as terracing and trenches with a width of $0.4 \mathrm{~nm}$ between deposited molecules. Further developments have employed in-situ analysis. With this technique thin films were produced utilising pulsed laser deposition within the STM chamber. The film growth was then characterised at an atomic sale without the inhabitation of containments [24], [25], [26] [27]. 

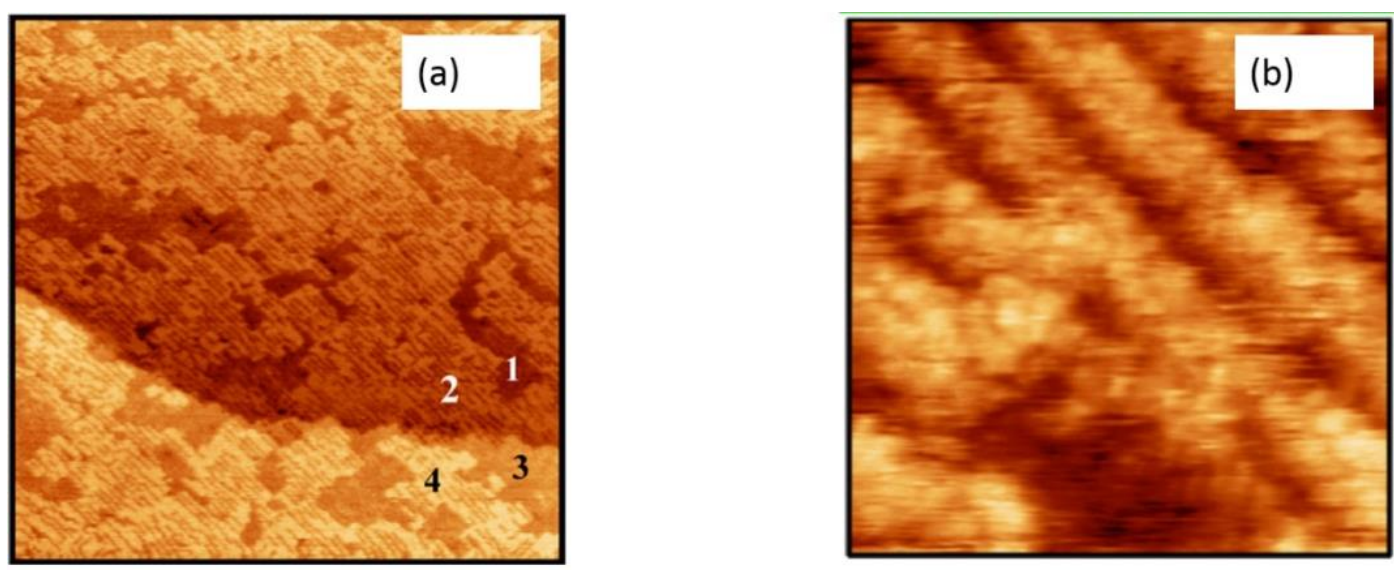

Figure 3.4 STM images of multilayer growth of APT on an iodine doped Au layer showing the

(a) multilayer growth across $130 \times 130 \mathrm{~nm}^{2}$ and (b) at high magnification, showing $8.1 \times 8.1 \mathrm{~nm}^{2}$ area, the formation of terracing and trenches [3].

Rafati et al (2017) utilized a NAMA-STM SS-3L1 to characterise gel extracted DNA nanotubes. The measurements were taken using a platinum-iridium tip, with the STM in constant current mode using a current set point $0.1 \mathrm{nA}$, sample bias voltage $0.2 \mathrm{~V}$ and low scan rate. Unlike previous work the images were captured under ambient conditions, without the requirement of high vacuum. The data was post-processed sequentially by line using averaging filters within the NAMA-STM analysis software. High resolution three-dimensional and two-dimensional images of elongated nanotubes were attained, as seen in figure 3.5. The images suggested the tubes had a height of 46nm from the topographical surface. The authors proposed STM as a viable technique for ultrastructural characterisation of DNA nanotubes and they suggested that the helical structure of the tubes can be determined within the images. 


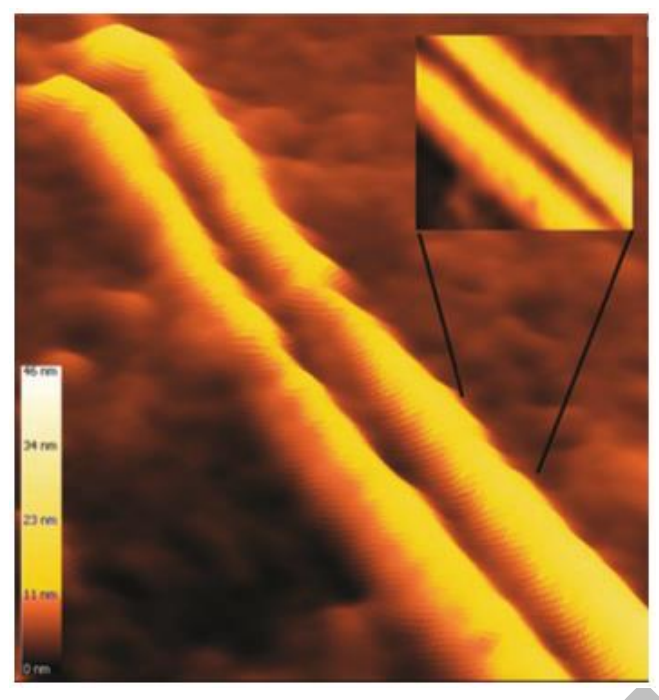

Figure 3.5 High resolution STM 3D micrograph image of DNA nanotube with 2D insert showing higher magnification of the structure details [28].

\subsubsection{Atomic Force Microscope (AFM)}

The Atomic Force Microscope (AFM), also called the Scanning Force Microscopy (SFM), was developed in 1986, subsequent to the STM. Similar in operation to the STM, the AFM involves scanning a sharp tip across a sample surface while monitoring the tip-sample interaction to allow the reconstruction of the three-dimensional surface topography. A typical AFM has nanometer lateral and sub-angstrom vertical resolution and can image insulators as well as conductors. UHV AFM resolution is comparable to that available from STM and the Transmission Electron Microscopy (TEM). An AFM consists of a sharp tip on the end of a flexible cantilever which is moved across a sample surface by piezoelectric actuators. The cantilever is typically made from $\mathrm{Si}$ or $\mathrm{Si}_{3} \mathrm{~N}_{4}$ with a tip curvature radius of a few nanometers. Displacement of the tip is recorded by a non-contact laser displacement measurement. A laser light directed onto the cantilever above the laser tip is recorded on a photo-detector area which allows calculation of displacement via signal strength measurement or triangulation. A feedback loop maintains a constant tip-surface 
interaction force by vertically moving the scanner to maintain a constant photodetector difference signal. The distance the scanner moves vertically is recorded at and with each $\mathrm{x}, \mathrm{y}$ position which allows the surface information to be presented and analysed. A complicated set of forces can be present at the tip-sample interaction. When the tip touches a surface under ambient conditions, a repulsive force is present. When the tip is at a small distance from the surface, attractive forces can be present as well as van der Waals forces and capillary forces arising from condensation of water vapour in the contact area. Operating modes can be roughly classified as contact, non-contact or dynamic.

In contact mode, the scanning tip is dragged across the sample surface and the tip deflection monitored. Using Hook's law, the force between the tip and the surface is automatically kept constant during scanning (typically between 0.1 and $100 \mathrm{nN}$ ). Lower stiffness cantilevers (spring constant, $\mathrm{k}<0.1 \mathrm{~N} / \mathrm{m}$ ) are used in this mode to amplify the deflection signal. Contact mode may not be suitable for soft materials which can be easily deformed or damaged, such as for polymer or molecular imaging. When scanning is performed in the region where the tip is attracted to the surface, the scanning is termed non-contact mode. In this region, the cantilever bends toward the sample. If an oscillatory tip displacement is sufficiently large to pass through both regions, the probe experiences both attractive and repulsive forces. This mode is known as dynamic, intermittent or tapping mode. Tapping mode was developed for investigation of soft materials [29]. In this mode, the cantilever oscillates near its resonant frequency and lightly taps the surface during scanning. The tip rapidly moves in and out of the sample surface with an amplitude that is sufficiently high to overcome adhesion forces so that it stays in contact only for a short fraction of the oscillation period. Depending on the cantilever type, the frequency typically varies from 50 to $500 \mathrm{kHz}$ and amplitudes up to $100 \mathrm{~nm}$ are used. The laser spot deflection is used to measure the 
amplitude of cantilever oscillation and a feedback loop maintains a constant oscillation amplitude by adjustments to the servo which adjusts the cantilever height.

\section{AFM case studies}

Ahad et. al introduced nanostructures on the surfaces of polymeric biomaterials by Extreme Ultraviolet (EUV) technology for biomedical engineering applications [30]-[35]. EUV photons are high energy radiations (from $10 \mathrm{eV}$ up to $124 \mathrm{eV}$ ) with nanometre wavelengths (124 nm to 10 $\mathrm{nm})$, therefore nano- surface structuring is possible. The surfaces of polytetrafluoroethylene (PTFE) polymer samples were irradiated with 50, 200 and 300 EUV pulses. The EUV exposed PTFE samples were investigated by AFM and images were acquired at two different resolutions $(50 \mu \mathrm{m} \times 50 \mu \mathrm{m}$ and $25 \mu \mathrm{m} \times 25 \mu \mathrm{m})$ to visualise the effect of EUV irradiation on the surface morphology. In order to establish the relationship between number of EUV pulses irradiated on the sample and the resulting surface structure, the cross-section analysis and histograms of the AFM images were obtained. The AFM images of pristine and EUV irradiated (300 pulses) of PTFE samples are present in figure 3.6 (a) and 3.6 (b), respectively [31]. The EUV surface modification resulted in formation of ripple-type regular structures on the PTFE samples. It was observed that with increasing number of EUV pulses, the surface roughness of the PTFE samples were increased and more high-rippled structures were formed on the sample surface. 

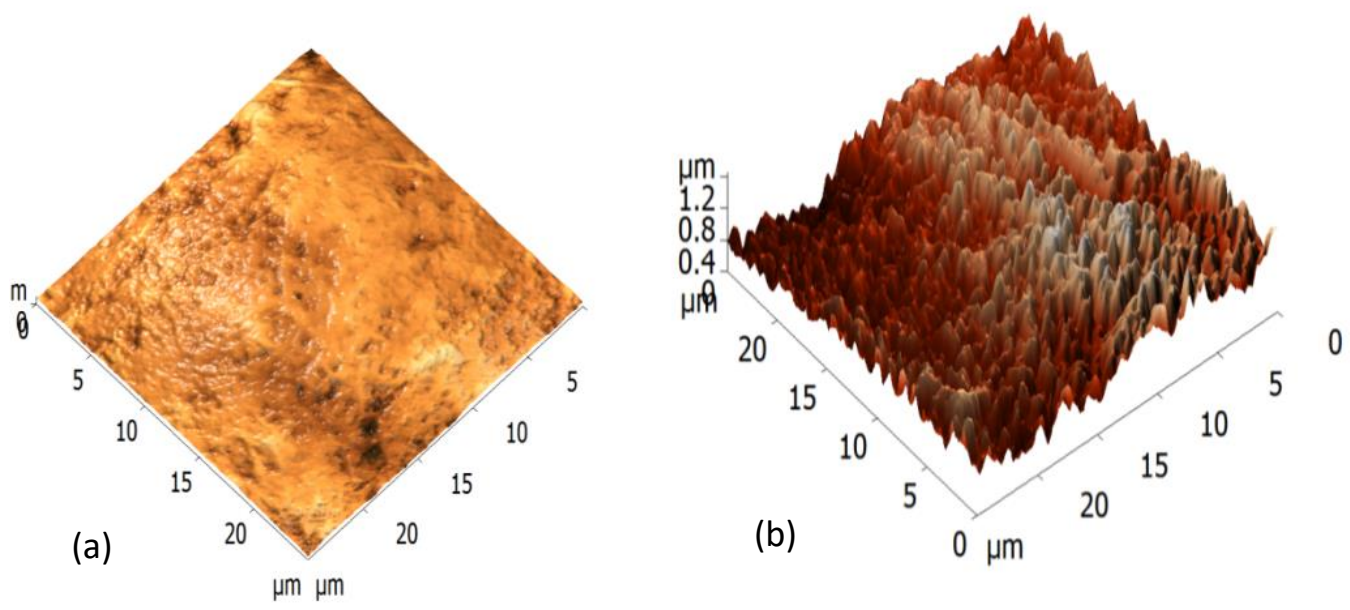

Figure 3.6 (a) AFM image of pristine PTFE sample and (b) AFM image of PTFE sample irradiated with 300 EUV shots [31]

The surface structuring and wettability control of PET films are often associated with biocompatibility control for biomedical engineering applications [30], [34]. In another study, Ahad et. al used the same EUV source to perform surface modification of polyethylene terephthalate (PET) polymer films in order to investigate the effect of surface structuring on wettability control [33]. The polymer films were irradiated in helium and nitrogen environments with 20 and 30 EUV pulses. AFM was used to measure the surface roughness and water contact angle measurements were taken to examine the changes in wettability behaviour. The EUV surface modification resulted in the formation of nano- and micro-structuring on the polymer surfaces. The results demonstrated that the average surface roughness of PET polymer films was increased from $6.6 \mathrm{~nm}$ to $234 \mathrm{~nm}$ and $271 \mathrm{~nm}$ when EUV processed in nitrogen and helium gases respectively. The surface structuring consequently increased the water contact angle making the PET surfaces more hydrophobic. It was observed in this study that for EUV-treated PET polymer 
surfaces, a direct relationship exists between surface roughness and hydrophobicity for EUVmodified PET samples, see figure 3.7.

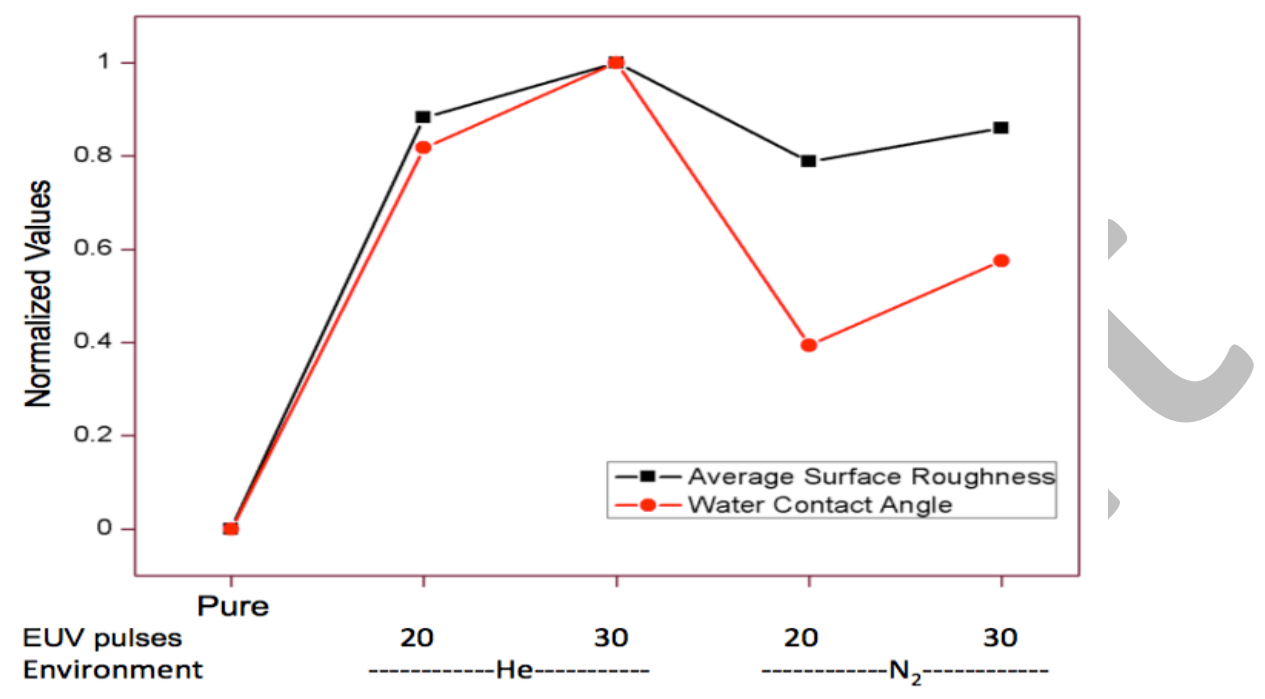

Figure 3.7 Normalized average surface roughness and water contact angle of pristine and EUV modified surfaces [33].

\subsubsection{Optical microscopes}

\subsubsection{Confocal microscopy}

In confocal microscopy two focusing arrangements are used to focus on the point in a sample to be imaged. One focuses laser light through an objective lens to the point of interest and the other focuses the reflected light to the imaging sensor. Light from the point to be imaged is passed through a pinhole such that all extraneous out of focus light is removed. This allows lateral resolutions approximately 1.4 times greater than in conventional microscopes to be achieved with confocal microscopy. The depth of the focal plane depends on the specimen optical properties and importantly on the squared value of the objective lens numerical aperture. Three dimensional reconstructions of cells and surfaces can be achieved with this technique. To achieve this, the 
sample is scanned such that one 2D slice is recorded, the focal plane of the sample is then moved a prescribed amount where the next $2 \mathrm{D}$ slice is recorded and this sequence is repeated until the required volume is scanned. Image processing software is then used to process the collected data to reconstruct the 3D object. Confocal microscopes are most often used to image biological systems and semiconductor surfaces [18-20].

Three variations of scanning are available in confocal microscope systems. In the conventional confocal laser scanning microscope the sample is raster-scanned which results in a scanning rate of about three frames per second. Such systems provide the highest spatial resolution, however for higher temporal resolution, the spinning disk (Nipkow) and the Programmable Array Microscope (PAM) systems can provide rates of 30 frames per second [36]. In a Nipkow disk system, a thin disk with hundreds of spirally patterned pinholes is spun in the light path to the objective lens. The pinholes only allow perpendicularly oriented rays of light to penetrate which allows high scanning speeds independent of the laser scanning speed. PAM is a variation on this whereby an acoustoor electro-optical filter can be patterned to automatically produce the pinhole pattern required. Such a system can allow for up to 1000 beams to simultaneously scan the entire field at millisecond scan speeds. High frequency scanning has the added advantage of reducing exposure of sensitive samples to photons which may cause damage due to photobleaching or phototoxicity.

Fluorescent dies are often added to a surface or flurophores to cellular systems to enable enhanced imaging with confocal fluorescent microscopy. Various excitation laser wavelengths are available for these systems ranging from 442 to $647 \mathrm{~nm}$ depending on the flurophore excitation and emission wavelengths used. Reflected and fluoresced light waves are emitted from the sample. A beam splitter can be selected to reflect only the fluoresced light to the detector which provides enhanced 
signal-to-noise ratio. As described above, the pinhole is also used in this set-up to eliminate the out of focus signal and record only the light from the region of interest.

\section{Confocal microscopy case studies}

In a recent study by Ilcíková et al. (2016), untreated and polystyrene modified single and multiwall carbon nanotubes (CNT) were added into a polymer matrix to produce a nano-composite polymer. These nano-polymers have been shown to possess increased functionality via improved mechanical and electrical properties. However, a significant challenge to the adoption of polymer nano-composites is ensuring an even distribution of CNTs within the matrix. In this work the authors address the issue by utilizing confocal laser scanning microscopy (CLSM) to characterize CNTs dispersed in the matrix. CNTs with a maximum thickness of 25-60nm were visualized both under reflection and fluorescent mode with the addition of benzothioxanthene fluorescent labelling dye to the polymer for the latter. Figure 3.8 shows the confocal images captures of the CNT nanocomposite matrix under both florescence mode and reflectance mode.
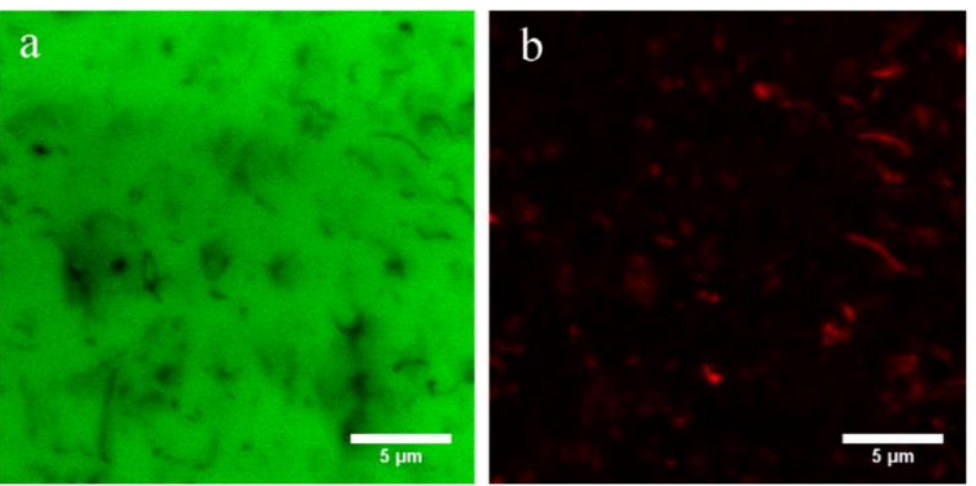

Figure 3.8 CLSM visualisation of CNT Nano-composite matrix under

(a) florescence mode and (b) reflectance mode (b) [37]. 
From the imagery, it was observed that smaller thickness (8-18nm) multi-wall CNTs were visualised only under fluorescent mode as can be seen in figure 3.9 (a-e). Thus, the fluorescent mode can be a useful and potentially a preferred approach to characterise nanocomposite structures. The CLSM in reflectance mode is as a non-invasive method to determine the dispersions of the CNT through the depth of the polymer by imagining at various z-axis positions and combining slice images into a 3D dispersion profile. This was completed for one micrometre slice depths as shown in figure 3.9 (f). CLSM was therefore demonstrated as a non-invasive technique to monitor the distribution of CNT both on the surface and embedded in the bulk material and offers a solution to bring quality monitoring into nano-composite fabrication.
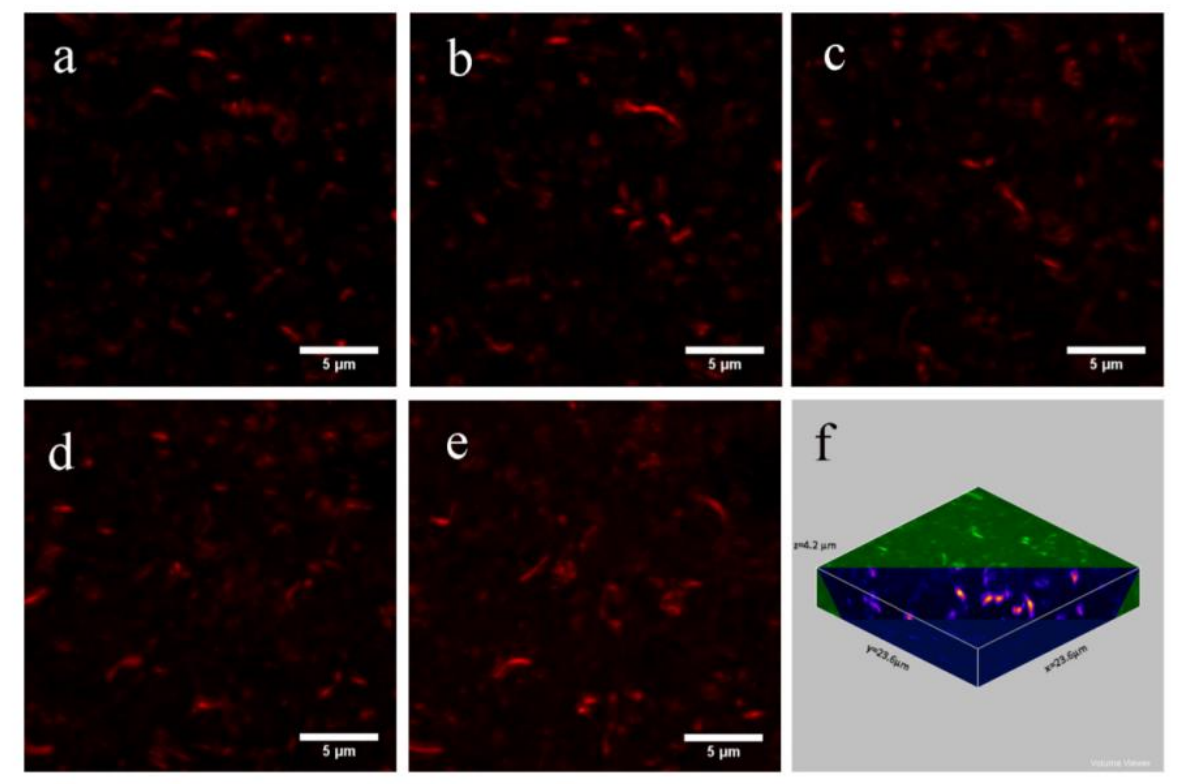

Figure 3.9 Optical slice images of polymer matrix (a-e) at various depths and (f) as a combined 3D image [37].

In other work, Oyarzún et al. (2015) employed 3D confocal microscopy to measure the thickness of $\mathrm{TiO}_{2}$ nonporous films by examining various height profile points on the sample surface. The authors suggest 3D confocal microscopy as their preferred technique compared with FESEM for 
thickness measurement of $\mathrm{TiO}_{2}$ layers as larger measurement areas can be achieved compared with more local results attained from FESEM [38].

\subsubsection{Near Field Scanning Optical Microscopy (NSOM)}

NSOM can be classified as a SPM technique as the scanning methodology places it in this category. However due to the wave based principles behind its operation, it is placed under the optical microscopy section within this chapter. When electromagnetic radiation is emitted, the near field is that part of wave front that is within a couple of wavelengths from the surface of the emitter. An evanescent wave is a near field standing wave exhibiting exponential decay with distance. Evanescent waves are strongest within one-third wavelength $(\lambda)$ from an electromagnetic emitter. Near Field Scanning Optical Microscopy (NSOM or SNOM) is a surface imaging technique with nanometre resolution.

The main components of a NSOM are: the scanning tip, the feedback mechanism, and the piezoelectric actuation and the light source. The light source is critical and is composed of a laser beam focused into an optical fibre via a polarizer, a beam splitter and a coupler. The polarizer and beam splitter removes additionally reflected light from the returning reflected light. The scanning probe is generally a sharpened optical fibre. The tip displacement is measured and the reaction normal force is recorded, to ensure the probe is at the correct height at all times. Alternatively, a tuning fork attached to the fibre tip is oscillated at resonant frequency which moves the tip laterally. Changes in amplitude are monitored to provide shear-force feedback.

\section{NSOM case studies}

Beleites et al. (2012) found that they could use NSOM to analyse the shape of a single Ag nanoparticle that had been post processed by femtosecond laser irradiation. Commercially 
available spherical silver nanoparticles suspended in an aqueous solution with mean particle diameter of $40 \mathrm{~nm}$ was drop-cast onto a substrate and dried under ambient conditions. Following that, the samples were covered with a $40 \mathrm{~nm}$ aluminium layer via atomic layer deposition. The samples were irradiated using a frequency-doubled Yb:KGWlaser system with $300 \mathrm{fs}$ pulse length, and then annealed for 60mins. Measurement was performed using an aperture-type cantileverprobe-based system in transmission mode.

The topography of the Ag nanoparticle, distinct to the aluminium oxide layer, was observed using a laser source with wavelength of $458 \mathrm{~nm}$, see figure 3.10. Along with topography the relative transmission was also able to be calculated by normalising the measured intensity compared with the background intensity. This technique offers promising abilities to characterise nanoparticle profiles while deposited on substrates.

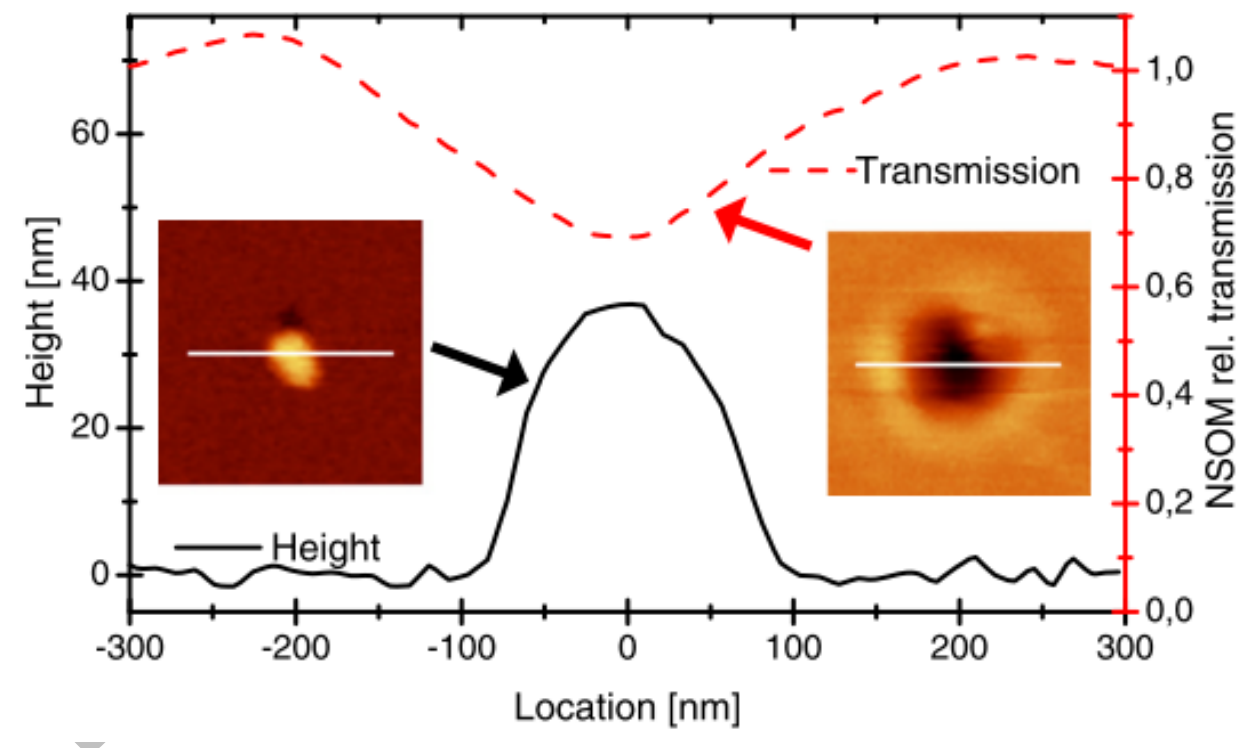

Figure 3.10 Characterisation of single Ag nanoparticle topography and relative transmission intensity excited at $485 \mathrm{~nm}[39]$. 


\subsubsection{Dynamic light scattering (DLS)}

Dynamic light scattering (DLS) techniques have been adopted as the primary on-the-bench method for measurement of nanoparticle size distribution in liquid suspensions. DLS is widely used in research and industry, it provides rapid size analysis of nanoparticle colloids. It has attained such market coverage that many nano-colloid researchers rely on it as their sole size characterisation technique. The technique is used for particle analysis in inks, paints, and more recently biological diagnostics and disease treatment systems [40], [41], indeed many fields where nanoparticle size is critical. The technique offers possibilities of in-situ measurement, which can be important for time sensitive process and industrial manufacturing.

Unlike microscopy techniques, the size measured is not just a function of the material core size in air, but the hydrodynamic diameter; including effects from fluid stabilizers, surfactants, and the electrical double layer thickness [42] while also providing an overall size distribution of the bulk colloid. Thus, the DLS offers a more representative measurement of NPs size while in the liquid phase. This may provide more useful data if the nanoparticle applications are in liquid suspension. Generally, the size distribution measured by DLS is larger than for microscopy techniques such as TEM and SEM where Nano colloids must be transferred to a substrate and dried. During this process the hydrodynamic properties are lost and drying aggregation may occur. It should be noted that the sample size of drop-cast particles for TEM and SEM is smaller compared with the "bulk" measurement of DLS. However there are factors which effect the techniques precision, due mainly to its base technology, how light interacts with differing size particles [43], leading to measurement masking effects. DLS operates on the principle that the diffusion coefficient (D) of a particle is size dependent where the Stokes-Einstein equation [43] describes the diffusion coefficient as:

$$
D=\frac{K_{B} T}{6 \pi \eta R_{S}}
$$


with $K_{B}$ being the Boltzmann constant, $T$ the colloid temperature, $\eta$ the solvent viscosity and $R S$ the sphere radius. This basically describes that light scatters at different rates with differing particle sizes [44].

During its operation samples are irradiated with a coherent monochromatic polarized laser beam light source, as seen in the figure 3.11. A scattering detector, positioned typically at an angle of $173^{\circ}$ backscatter [45] to the beam collects the dispersed pattern containing intensity fluctuations of light. This dispersed light intensity pattern is due to constructive and destructive inference on different areas of the detector [46]. The scattering of light is related to the position of particles in the sample and the scattered light from each particle will be in its unique phase while striking the detector surface. This scattering intensity is a function of: particle size, solute-particle concentration, refractive-index difference of solvent and solute particles [47]. Therefore, if measurements are repeated at known time intervals, the intensity change of the scattered patterns over time is accounted for by Brownian motion of particles and the Intensity change can be related to the diffusion coefficient and particle size.

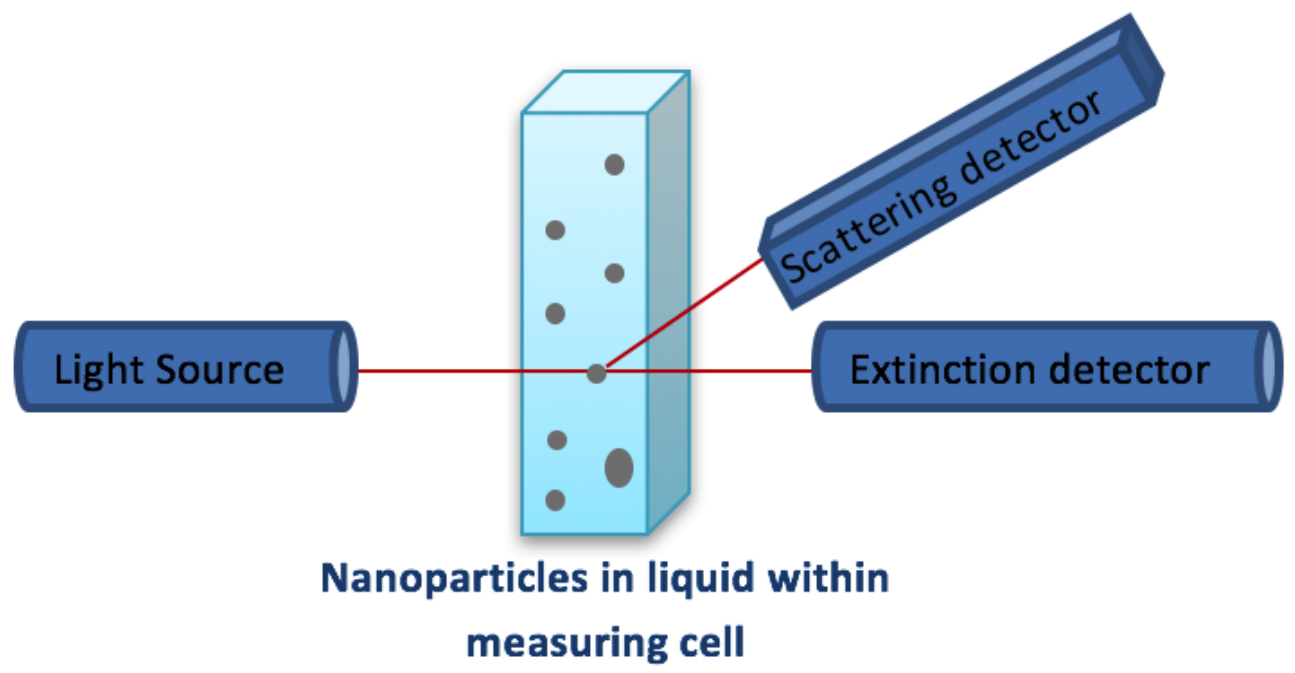

Figure 3.11 Schematic showing DLS operation, including coherent monochromatic polarized light source, extinction detector and scattering detector placed at a defined incident angle. 


\section{Case studies}

Tomaszewska et al. (2013) investigated the detection limits of DLS for the characterisation of polydisperse nanoparticle colloids. To make a controlled, artificial polydisperse colloid, the authors mixed known concentrations of standardly produced and characterised $10 \mathrm{~nm}, 55 \mathrm{~nm}$ and 80nm Ag nanoparticles, confirming their measurements with AFM and TEM. They found that the polydispersity of the colloids was problematic to measurement of the true colloid size, as the light scattering intensity from the larger NPs masked out the smaller particles. The authors concluded that as low as a 5\% presence of larger NPs in the sample population was enough to mask the detection of the smaller nanoparticle colloid, making up 95\% of the sample population [42]. This would certainly raise question marks over the validity of use of DLS have highly polydisperse populations, and leads to problems when considering aggregates in the colloid.

Horechyy et al. (2017) utilised in-situ DLS to monitor the growth of silica core shells on copolymers. The authors used disposable DLS cuvettes to hold the co-polymer mixture. Silica sol was added to PS-b-P4VP micelles, mixing the reaction mixture for 2 mins before placing the cuvette into the Malvern Zetasizer Nano S and starting $\mathrm{Z}$ measurements at 2 min intervals. The refractive index and fluid viscosity was set for standard methanol. Each measurement 100s utilising ten auto-correlations with a 10s scan speed. The data was averaged using Dispersion Technology Software (DTS). Using this technique, the authors observed five distinct stages in silica shell formation [48]. Time dependent particle diameter for the initial stage after the addition of $\mathrm{SiO} 2$ is illustrated in figure 3.12. The authors found that TEM and DLS measurements of the growths stages showed contradictory trends, where within the first hour a dramatic decrease in particle size was observed on the DLS, and not so much with the TEM. Also after several days 
there was an observed increase in the DLS particle size measurement, not evident from TEM analysis. After further inspection the cause of these discrepancies was determined as follows:

1. The increasing density of the silica shell lead to a reduction in the hydrodynamic particle size observed by the DLS.

2. After several days, the hydrodynamic particle size increased due to changes in the fluid viscosity, but the shell growth was substantially smaller.

In this work the authors utilised in-situ DLS measurement, concluding that in-situ monitoring was a useful tool in understanding the full growth profile of silica shell nanoparticles.
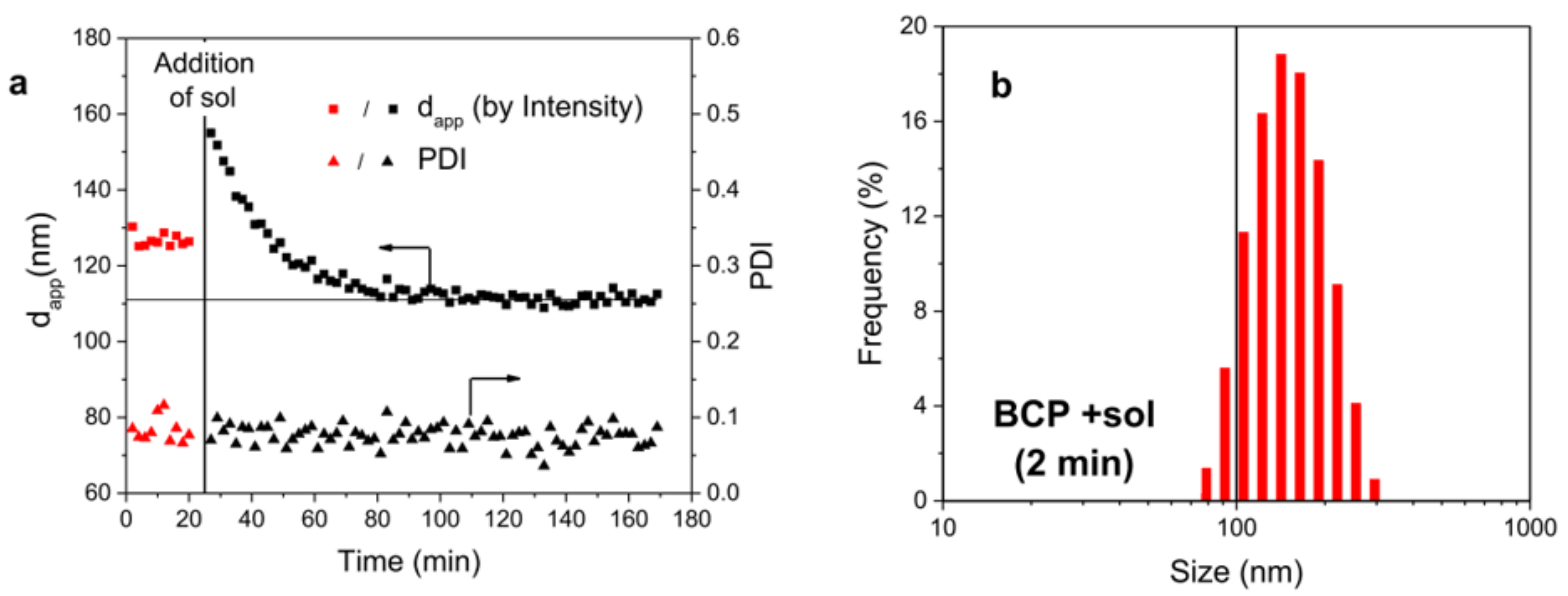

Figure 3.12 (a) Apparent particle size (squares) and PDI (triangles) of PS-b-P4VP co-polymer before (red) and after the addition of $\mathrm{SiO} 2$ (black) over time showing silica shell evolution; and (b) intensity of particle size distribution, 2 mins after the addition of $\mathrm{SiO} 2$ [48].

\subsubsection{Measurement of nanostructures internal geometries}

\subsubsection{Transmission Electron Microscope (TEM)}

TEM is an established characterisation technique, which can provide both image mode and diffraction mode information from a single sample [49]. It is regarded as one of the main techniques for nanomaterials characterization, largely due to its high lateral spatial resolution in 
the region of $0.08 \mathrm{~nm}$ [50]. A feature of nanomaterials is that specific properties, for example, colour, can be related to a particles size. Agglomeration of nano-particles, or failure to isolate individual nano-structures is likely to result in anomalous property characterisation. Characterising the elastic or mechanical properties of individual nanoparticle/nanotube/nanofibers is a challenge to many existing testing and measurement techniques. It is difficult to pick up samples and difficult to clamp samples, in order to test for tensile strength or creep, for example [51].

The modern TEM are capable of formatting nanometre size electron probes having diameters ranging from $2 \mathrm{~nm}$ to $5 \mathrm{~nm}$. This formation is possible by employing a multistage condenser lens system. This lens system makes scanning transmission mode possible and the resulting electronprobe diameter defines the resolution of the system. Therefore, in addition to thin samples, specimens with higher degree of crystallinity and thickness can be imaged by TEM. Multistage condenser lens systems enable recording of secondary and backscattered electrons. This has advantages for imaging thick or crystalline specimens and for recording secondary electrons and backscattered electrons. The inhomogeneity in cathodoluminescence can also be recorded using complex multistage condenser lens system for correlation with structural defects [52]. Cathodoluminescence microscopy is a useful characterization technique in various fields related to optoelectronics, energy, geology, cellular biology, and healthcare [53]. Traditionally Scanning Electron Microscopy (SEM) has been used to study the cathodoluminescence of bulk samples as well as nanoparticles. The limited resolution of SEM (up to $20 \mathrm{~nm}$ in the most advanced systems) restricts the use of SEM for microstructure correlation with the cathodoluminescence. Therefore high resolution cathodoluminescence microscopy is possible using TEM [54]-[57]. 


\section{TEM case studies}

TEM has shown itself to be capable of meeting such challenges. It is commonly used specifically for its ability to isolate and examine individual nanoparticles. This approach reduces the potential for agglomeration which can be a problem with wet-based laser scattering techniques. Nanoparticles of various materials produced by different techniques have also been imaged, with remarkable clarity [58]-[61]. TEM's electron imaging and diffraction options allow propertystructure relationships of nano-structures to be understood. It has the resolution to differentiate between nanotubes with subtle nano-scale structural patterns. Interlayer distances of about 0.34 $\mathrm{nm}$ have been measured and imaged with impressive clarity, consistent with the (002) plane lattice parameter of graphited carbon [62].

The phase transformation and degradation in the nanostructure of the Si during the lithiation process in the lithium ion batteries results in $\mathrm{Si}$ amorphization to $\mathrm{Li}_{\mathrm{x}} \mathrm{Si}$ ph ase. The detailed mechanism of this process is not yet fully understood and in-situ nano-characterisation techniques are required to investigate the charging and discharging processes. TEM provides a unique characterisation platform to investigate the fine details of complex mechanisms, impossible to study otherwise. Wang et. al reported the characteristics of the phase transformation and the variations in the microstructure properties of amorphous $\mathrm{Si}$ - coated hollow carbon nanofiber (CNF) composites [35]. The silicon/carbon nanofiber composite was used to coat the interior and exterior of a nanobattery anode. The in-situ TEM charging and discharging of the carbon nanofiber composites was performed. The spontaneous crystallisation of $\mathrm{Li}_{15} \mathrm{Si}_{4}$ from the amorphous $\mathrm{Li}_{x} \mathrm{Si}$ was observed. Contrary to classic nucleation and growth progress the corresponding phase transition process was observed without phase separation or large-scale atomic motion. The coating of amorphous $\mathrm{Si}$ layer on the $\mathrm{CNF}$ was strong in the starting charge/discharge cycles 
without any structural defects. However, with increasing charge/discharge cycles, the bond between amorphous $\mathrm{Si}$ and CNF weakens as the surface roughness is increased, see figure 3.13. This degradation of the nanostructure observed by in-situ TEM eventually reduces the capacity of the composite anode over extended period of charge/discharge cycles.
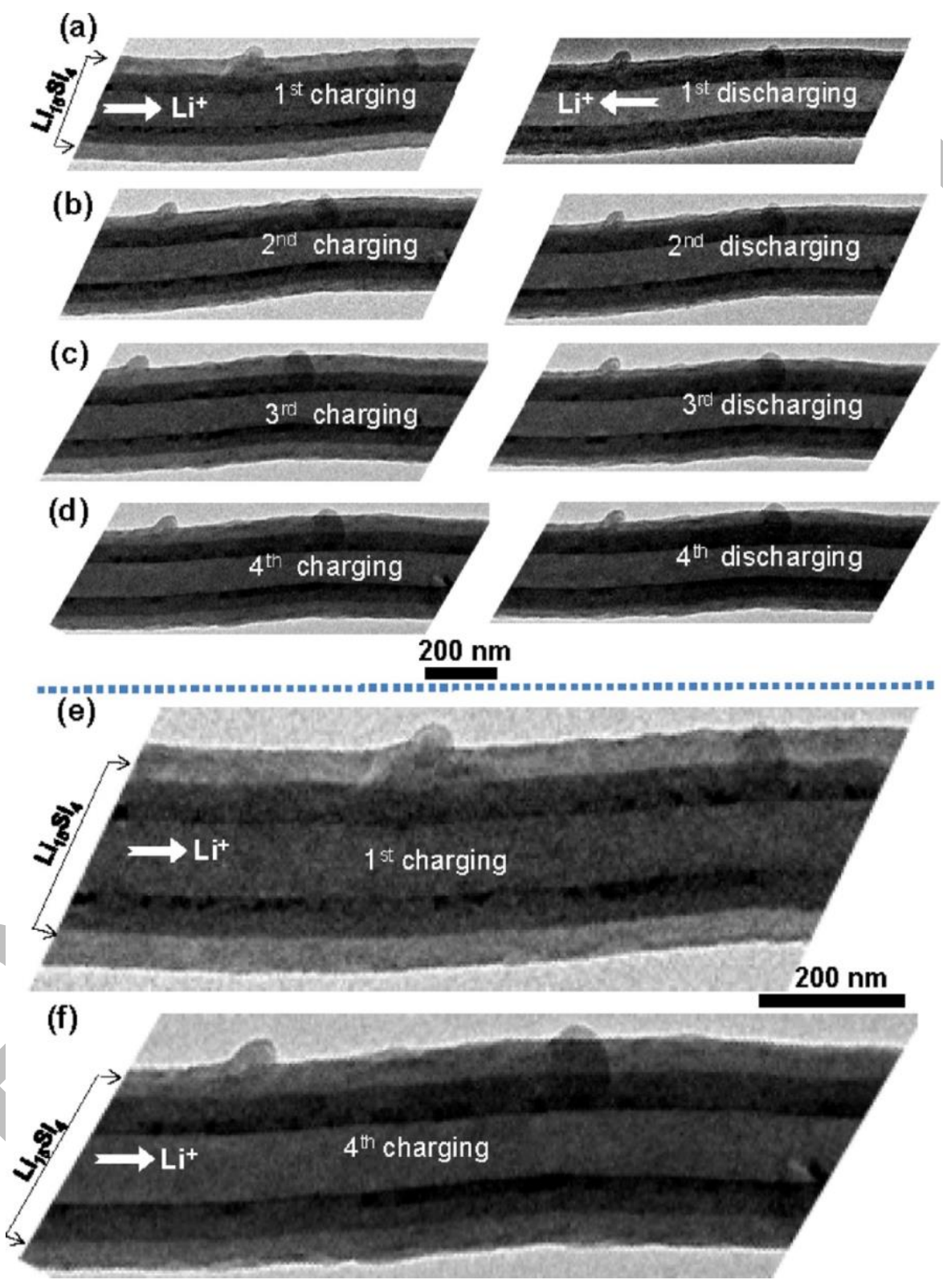

Figure 3.13 TEM images showing the structural evolution of Si-CNF during the cyclic charging and discharging, showing $(\mathrm{a}-\mathrm{d})$, the left column is charged and the right column is discharged; and (e) a high magnification image of (a); and (f) a high magnification image of (d). 
Note that with cyclic charging and discharging, the surface of the coating layer is gradually crumpling. The two particles shown in this CNF are pre-existing particles. With a very limited number of in situ cycling in the TEM column, the change of the coating layer is not significant. However, as a general trend, it is noted that even with a limited number of cyclic charging/discharging, the surface begins to become more rough. To illustrate this point, the images in figure 3.13 (a) 1st charged and figure 3.13 (d) 4th charged are shown magnified in figure 3.13 (e) and figure 3.13 (f) respectively. Note the slightly increased surface roughness in (f) as compared with (e) [63].

The in-situ observations of other nanostructures and nanoparticles have been extensively study using TEM to highlight the fine details of many complex nano-scale mechanisms and processes [64]-[69].

\subsubsection{Focused Ion Beam (FIB)}

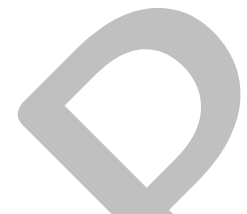

Focussed ion beams have become a popular tool for surface modification of materials and functional structure prototyping at the micro- and nanoscale. Modern focused ion beams have spot sizes of $<5 \mathrm{~nm}$ and are produced by using electrostatic lenses to focus the image of a point source, often gallium liquid metal ion source, onto the substrate and to deflect it in a precise fashion. For a comprehensive review of recent developments in FIB implantation and sputtering, FIB gasassisted etching, and FIB induced deposition the reader is referred to Stanishevsky [70] .

\section{FIB case studies}

Hitachi pioneered the use of FIBs for integrated circuit cross-sectioning for failure analysis in 1985. FIB technology can quickly and selectively remove specific layers (dielectric or metal) for 
conducting material analysis of underlying surfaces [70]. FIB can locate, expose and analyze the fault area without destroying the surrounding areas or losing the information at the site of the fault. A growing application area is FIB 'microsurgery' for circuit modification, device modification and defect repair. FIB make-and-break microsurgery combines imaging, restructuring, and verification in the same machine.

The provision of transport carriers, large surface area, and facile strain relaxation make nanowires a unique solution for various biomedical engineering applications related to biochemical sensing, electrophysiology, and intercellular activities recording. The cell viability on any substrate depends upon the interface activities between the substrates and cells. Despite extensive research on cellular interaction with nanostructured surfaces, the biological interactions between nanowires and biological cells are poorly understood. In a study by Wierzbicki et. al, low and high density nanowire (nanograss) silicon substrates were used to study the in-vitro 3T3 Fibroblast cell behaviour using SEM-FIB [71]. Four different types of interactions were observed between the nanograss and the cells: (i) the cells lying on the top of the nanowires, (ii) penetration of nanowires into the cells, (iii) the nanowires breaking from the substrate and becoming engulfed by the cells, (iv) weak nanowires being flattened by the cells, (v) cell motility through plasma membrane blebs, and (vi) Increased vacuolisation, see figure 3.14 [71]. 


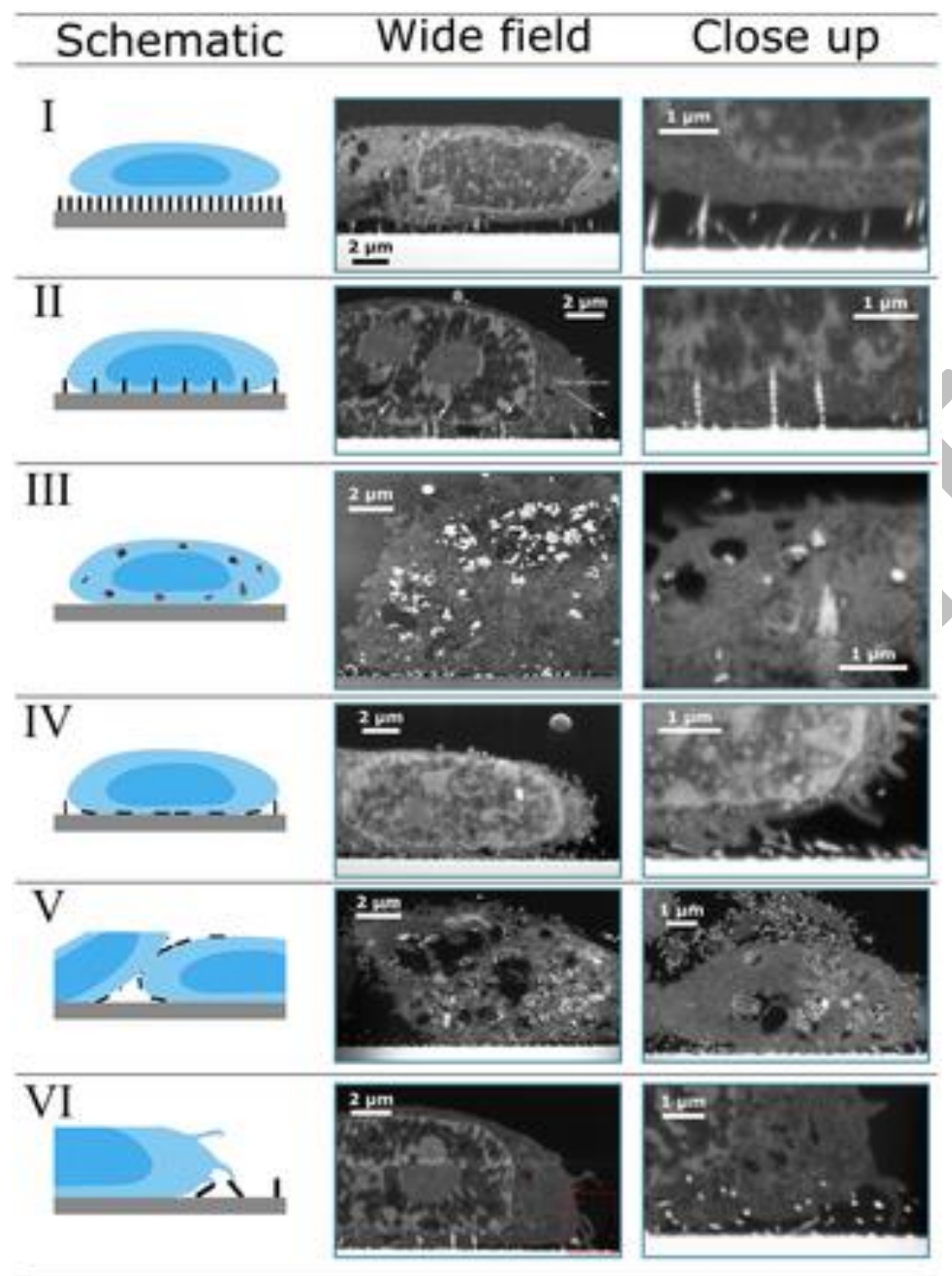

Figure 3.14 FIB-SEM images of several cases observed by cell - nanograss interaction with associated schematic description [71].

\subsubsection{X-ray diffraction (XRD)}

$\mathrm{X}$-ray Diffraction (XRD) is a powerful technique used to uniquely identify the crystalline phases present in materials and to measure the structural properties (strain state, grain size, epitaxy, phase composition, preferred orientation, and defect structure) of these phases. XRD is non-contact and 
non-destructive. $\mathrm{XRD}$ is most sensitive to high-Z elements; as a consequence, the sensitivity of XRD depends on the material of interest [49]. The regular array of atoms in a crystalline material forms a three-dimensional diffraction grating for waves with a wavelength close to that of the distance between the atoms. When waves enter a crystal, they are scattered in all directions by the atoms. In certain directions, these waves can interfere destructively. In other directions, constructive interference will occur resulting in peaks in X-ray intensity. The diffraction pattern that results is a map of the reciprocal lattice of the crystal and can be used to determine the structure of the crystal [72]. Bragg's law (3.2) is the basis for crystal diffraction:

$$
n \lambda=2 \mathrm{~d} \sin \theta
$$

where $n$ is an integer known as the order of diffraction, $\lambda$ the $\mathrm{X}$-ray wavelength, $\mathrm{d}$ the spacing between two consecutive scattering planes, and $\theta$ the angle between the atomic planes and the incident (and diffracted) X-ray beam [47].

\section{XRD case studies}

In a study by Liu et. al, graphene reinforced aluminium metal matrix composites (AMCs) were prepared and the characterisation of the AMCs was performed by SEM and XRD [73]. The reduced graphene oxide ( $\mathrm{rGO}$ ) was coated on the aluminium powder by mixing and dispersion using three different solvents (acetone, ethanol, and ethanol - water). The clearest dispersion of rGO and $\mathrm{Al}$ was observed in acetone solvent mixture. The GO-Al powders were prepared with $0.07 \%, 0.15 \%$, $0.3 \%, 0.7 \%$, and $2 \%$ graphene on a weight basis. Disc-shaped pallets of graphene- reinforced AMCs were compacted and sintered having $20 \mathrm{~mm}$ diameter and $0.8-1 \mathrm{~mm}$ diameter. The XRD scans for pure aluminium and graphene-reinforced AMC demonstrated major aluminium peaks at $38.8^{\circ}\left(\begin{array}{lll}1 & 1 & 1\end{array}\right), 45.0^{\circ}(200), 65.4^{\circ}(220), 78.5^{\circ}$ (3 11 1) and 82.7 (2 2 2), see Fig. 3.15. The presence 
of aluminium oxide was also observed at $27.1^{\circ}$ in all samples. The input variables such as graphene weight percentage, dispersion time, compaction pressure, and sintering temperature were optimized. The hardness test measurements demonstrated significant increase in hardness due to graphene nanosheets reinforcements. Graphene nanosheet characterisation has been performed using the XRD technique in various studies related to energy, mechanical properties improvement, and sensor development [74]-[78].

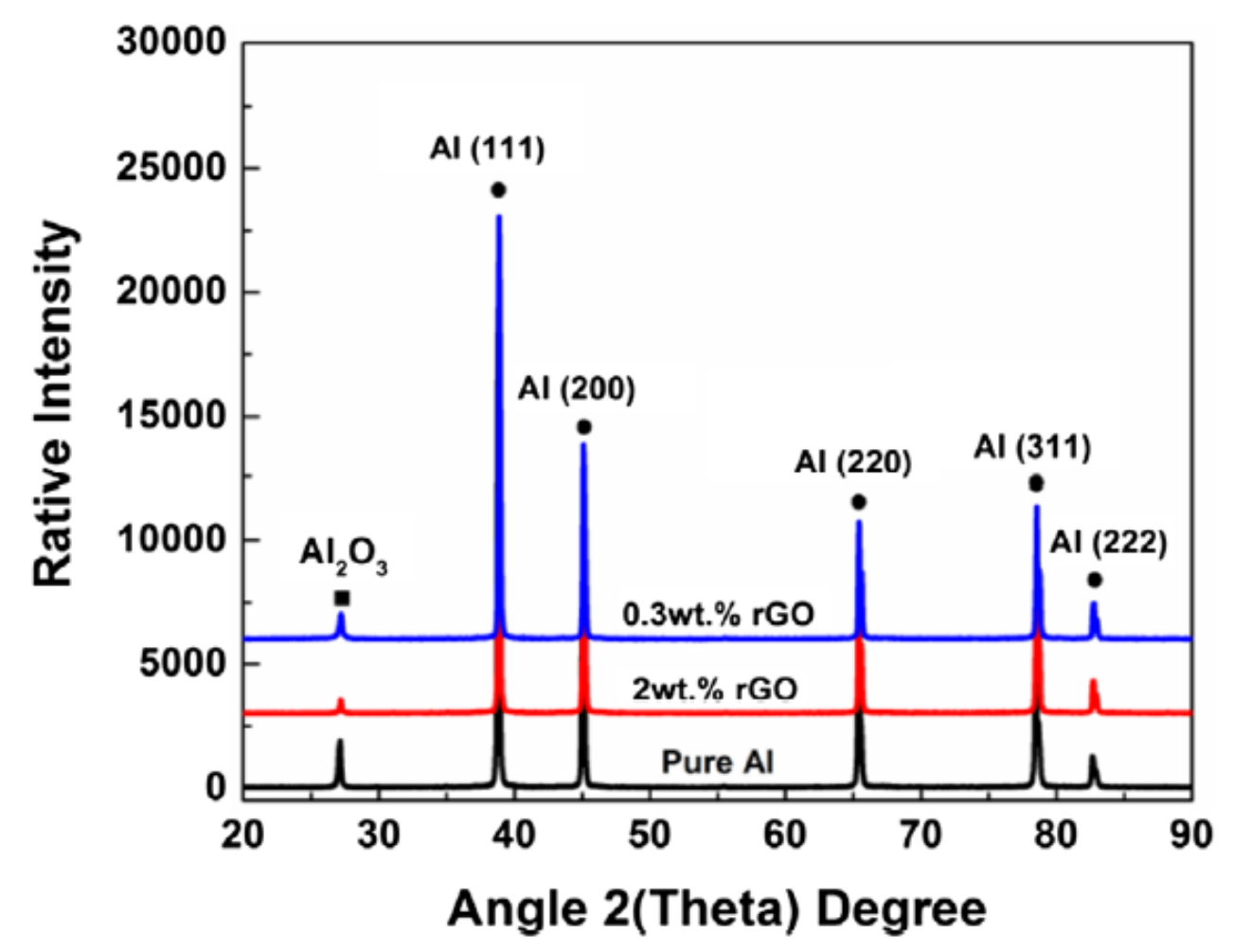

Figure $3.15 \mathrm{XRD}$ scans of pure aluminium, 0.3 and $2 \mathrm{wt} \%$ rGO-AMCs samples.

\subsubsection{Mercury Porosimetry}

The mercury porosimeter is a device which can generate suitably high pressures while measuring simultaneously both the pressure and volume of mercury taken up by a porous material [79]. 
Mercury does not wet most substances and will only penetrate pores when forced to do so under high pressure. Entry of mercury into pores requires applying pressure in inverse proportion to pore size. In other words, large pores will fill first, with smaller pores filling at increasingly higher pressures. Equation 2, known as the Washburn equation, is the basis of the mercury porosimeter method for measuring pore size distribution:

$$
D=\frac{-4 \gamma \cos \theta}{P}
$$

where $\mathrm{D}$ is the pore diameter, $\gamma$ the surface tension, $\theta$ the contact angle and $\mathrm{P}$ the applied pressure. Mercury exhibits a high contact angle against most solids. Reported contact angles vary, with $130^{\circ}$ being the most widely used value. Liquid mercury has a high surface tension; usually its value is taken to be $485 \mathrm{dyne} / \mathrm{cm}$ [80]. High-pressure mercury porosimeters can normally attain maximum pressures of 30,000 psia or 60,000 psia. The measurable pore size ranges from a maximum of $360 \mu \mathrm{m}$ to a minimum of $6 \mathrm{~nm}$ (for the 30,000 psia system) or $3 \mathrm{~nm}$ (for the 60,000 psia system) [80]. In principle, it would be possible to explore in the pore range below $3 \mathrm{~nm}$ if a porosimeter with sufficiently high pressures could be made [79]. In practice, BET surface area is normally employed for micropore $(<2 \mathrm{~nm})$ analysis. In porosimetry, a mercury filling apparatus is used to evacuate the sample and then to surround the sample with mercury. Evacuation is achieved by exposing the sample to a vacuum. The sample to be analysed is contained inside a penetrometer, which is a long glass capillary tube, the sample end being a bulb shape. Using the vacuum control on the filling apparatus, gases and vapours are removed from the sample. The vacuum valve is closed and the penetrometer so that the stem end is immersed in mercury. The vent control valve is then slowly opened so that air fills the mercury chamber. Mercury is forced 
up the capillary stem and into the bulb. The filled penetrometer is then removed and inserted in the high-pressure porosimeter for pore analysis.

The construction of the glass penetrometer is key to pore measurement. A metal sheath fits over the capillary section. A metal seal attaches to the sample end of the penetrometer as a base electrode. The construction is thus mercury-glass-metal, or conductor-insulator-conductor. In this way a coaxial capacitor is created [80]. The capacitance changes as a result of the change in mercury level within the penetrometer. The mercury level will change as porous samples are filled with mercury under increasingly high pressure. In a similar way, a normal mercury thermometer will change mercury level, indicating a temperature change. Some porosimetry systems operate on the basis of a wire dipped remotely into the mercury; the change in electrical resistance of the wire being used as a means of measuring the volume of mercury taken up by the pores [79].

\section{Mercury porosimetry case studies}

Terock et al. (2016) used mercury porosimetry to measure the pore size distribution of synthesized platinum-nickel nanostructures on porous zirconia, a material widely used in sensor and fuel cell applications including catalytic converters, where maintaining open pores is critical their operation [81]. An investigation into the mechanical properties and microstructures of fabricated nanocomposite aerogels using mercury porosimetry was conducted by Seraji et al. (2016). These nanocomposites were produced for applications in high temperature thermal insulation. Silicon carbide $(\mathrm{SiC})$ and carbon/silicon carbide $(\mathrm{C} / \mathrm{SiC})$ nanocomposite aerogels were fabricated via a thermal reduction process of high char yield precursors. The initial network structure of the precursor material is maintained during the reduction process, however the pore volume and size 
distribution along with mechanical surface properties are affected by the high temperature treatment process. These pore properties are directly related to the insulating properties of the material and therefore it is necessary to quantify them at the final processing step.

The pore and mechanical properties of novolac-silica hybrid polymer gels fabricated utilising the new solvent-saturated vapor atmosphere (SSVA) method was investigated in this work along with the polymer microstructure and variations of crystalline phase observed by porosity analysis over the course of the heat treatment process. Pore volume and size distribution were measured over a pressure range of $0.01-440 \mathrm{mPa}$ as can be seen by the intrusion curve in figure 3.16 . The sudden change in the slope of the volume versus pressure indicates a pressure transition point $\left(\mathrm{P}_{\mathrm{t}}\right)$. It was seen from the results for all materials tested that the initial pressure reduced with increasing amounts of silica. The authors found that with the addition of silica aerogel particles the pore size increased and the microstructure was directly proportional to the silica content.

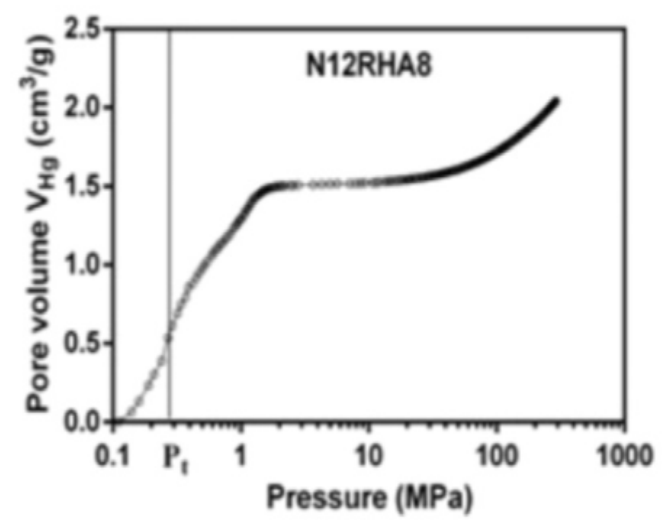

(a)

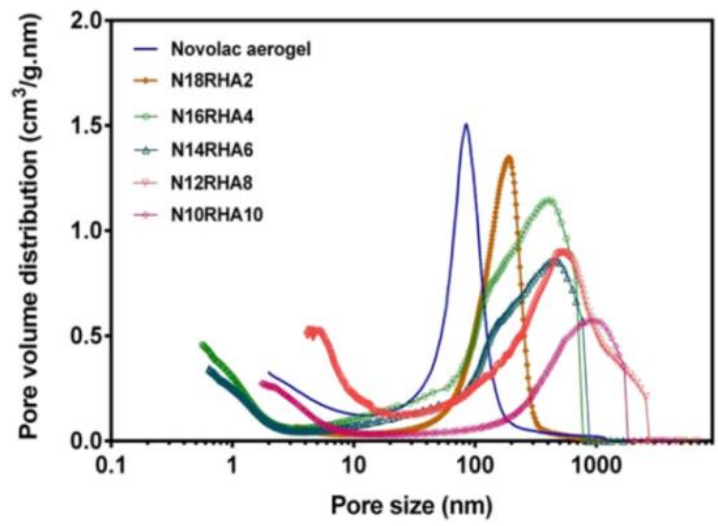

(b)

Figure 3.16 (a) Mercury intrusion curves of polymeric novolac/silica nanocomposite aerogels; and (b) pore size distribution of novolac aerogels[82]. 


\subsubsection{Measurement of composition of nanostructures}

\subsubsection{Energy dispersive X-ray spectroscopy (EDS)}

Energy dispersive X-ray spectroscopy is one of the most common spectroscopy techniques used as SEMs from the 1960's have been commonly equipped with this chemical analytical device [83]. In this technique, electromagnetic radiation is bombarded into a material surface which causes electrons from inner atomic shells to be ejected and subsequently filled with electrons from higher energy levels. Electromagnetic radiation used to excite the sample is usual a focused high energy stream of electrons, protons or X-rays. In a typical SEM, a stream of electrons is used. Electron transitions from the higher energy shells to lower energy shells causes X-rays to be emitted. A detector of SiLi or more commonly now a Silicon Drift Detector is used to collect and count the number of X-rays emitted at each energy level. The energy level characterises the element from which the X-ray was emitted while the count of the number of X-rays with this energy level is used to characterise the amount of the element that is present. A typical spectrum presents the count of the X-rays versus the energy level of the X-rays. New EDS systems come pre-calibrated to allow automatic detection and quantification of the elements present within the sample.

Care must be taken when interpreting EDS results. Wrong elements for example can often be detected where energy levels emitted from different elements overlap. X-rays can be generated from K, L, or M energy-level shells in a typical element. Therefore overlapping of energy levels detected can occur for example when Ti and $\mathrm{Ba}\left(\mathrm{Ti}-\mathrm{K}_{\alpha}\right.$ and $\left.\mathrm{Ba}-\mathrm{L}\right)$, or $\mathrm{Mn}$ and $\mathrm{Fe}\left(\mathrm{Mn}-\mathrm{K}_{\beta}\right.$ and $\mathrm{Fe}-$ $\left.\mathrm{K}_{\alpha}\right)$, or $\mathrm{Mn}$ and $\mathrm{Cr}\left(\mathrm{Mn}-\mathrm{K}_{\alpha}\right.$ and $\left.\mathrm{Cr}-\mathrm{K}_{\beta}\right)$ are present. Some knowledge of the sample elemental chemistry or knowledge gained from other analytical techniques is often useful for element 
identification. Wavelength Dispersive X-ray spectroscopy (WDS) is similar to EDS but analyses the diffraction patterns from the material-radiation interaction in order to identify one element at a time. WDS provide greater spectral resolution. Often the use of EDS followed by WDS can provide further definition of sample elemental content. The interaction volume from which X-rays are emitted due to the primary electron bombardment is in the shape of a tear drop beneath the surface. The accelerating voltage used and the density of the material define the volume size. The depth from which X-rays are emitted to the detector is usually from 1 to $5 \mu \mathrm{m}$ and can be calculated from the empirical expression $\left(0.1 \times \mathrm{E}^{1.5}\right) / \rho$ [84]. The width of the volume can be approximated from $\left(0.077 \times \mathrm{E}^{1.5}\right) / \rho$.

\section{EDS case studies}

The surface characterisation and texture analysis of nanostructured CMOS based MEMS devices is required to optimize the fabrication process and establishing the relationship between the mechanical and structural design of MEMS diaphragm and the selection of materials. In a study by Khan et. al, the surface chemistry of fourth generation CMOS-MEMS-based digital microphone chip was investigated by Carl Zeiss Ultra Plus SEM with an EDS detector [85]. The microphone chip was fabricated by integration of a diaphragm (MEMS) with a CMOS circuitry for signal processing. The EDS results demonstrated that the diaphragm was mainly composed of aluminium (approx $51 \mathrm{wt} \%$ ) covered by titanium layer (about $10 \mathrm{wt} \%$ ). A protective light coat of silicon oxide was observed, see figure 3.17. Traces of fluorine were also identified ( $2 \mathrm{wt} \%)$. These trace amounts could be introduced to the chip as by-product during the plasma etching process. The presence of fluorine-based silicon and aluminium radicals induce residual stress and strain on the diaphragm. The stress and strain produced on the diaphragm membrane influence the signal to noise ratio and 
sensitivity, thus reduce the overall efficiency of the chip. The elemental distribution of nanostructures [86]-[90], nanosheets [91]-[95] and nanoparticles [96]-[99] has been investigated extensively by EDS.

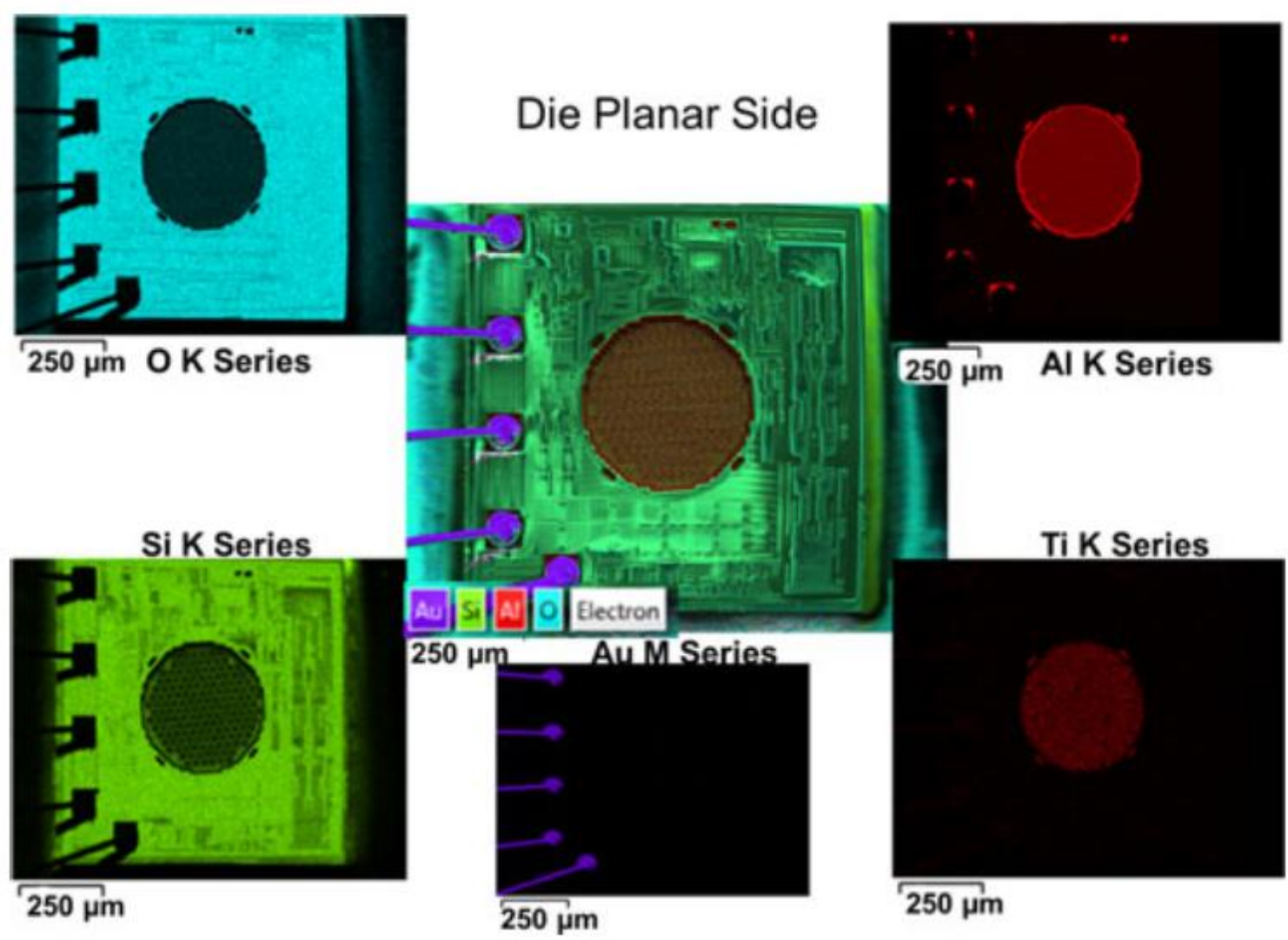

Figure 3.17 SEM-EDS on planar (top) side of the CMOS-MEMS die. O (Sky Blue), Al (Dark Red), Si (Light Green), Au (Indigo) and Ti (Light Red) [85].

\subsubsection{X-ray photoelectron spectroscopy (XPS)}

When excess electromagnetic energy is transferred to an electron that is in an outer shell it is called an Auger electron. An analysis of these electrons for chemical identification is known as Auger Electron Spectroscopy (AES). X-ray photoelectron spectroscopy (XPS) analyses electron emission of similarly high energy [100]. XPS can be used to measure the chemical or electronic state of 
surface elements, detect chemical contamination or map chemical uniformity of biomedical implant surfaces.

For XPS the material to be examined is irradiated with aluminium or magnesium X-rays. Monochromatic aluminium $\mathrm{K}_{\alpha} \mathrm{X}$-rays are normally produced by diffracting and focusing a beam of non-monochromatic X-rays off of a thin disc of crystalline quartz. Such X-rays have a wavelength of $8.3386 \AA$, corresponding photon energy of $1486.7 \mathrm{eV}$, and provide a typical energy resolution of $0.25 \mathrm{eV}$. Non-monochromatic magnesium X-rays have a wavelength of $9.89 \AA$, corresponding photon energy of $1253 \mathrm{eV}$, and a typical energy resolution of $0.90 \mathrm{eV}$. The kinetic energy of the emitted electrons is recorded. This kinetic energy of the ejected electrons is directly related to the element specific atomic binding energy of the liberated. A plot of these energies against the corresponding number of electron counts provides the spectrum which indicates the qualitative and quantitative elemental composition. At these higher energies, XPS only analyses to a depth of $10 \mathrm{~nm}$ into the surface. Electrons emitted at greater depths are recaptured or trapped in various excited states within the material. Spectral profiles up to $1 \mu \mathrm{m}$ deep can however be obtained by continuous spectral recording during ion etching or from consecutive ion etching and XPS measurement steps.

XPS is usually performed in UHV and typically provides resolutions down to $1000 \mathrm{ppm}$. With optimum settings and long recording times, resolutions down to $100 \mathrm{ppm}$ can be achieved. Nonmonochromatic X-ray sources can produce a significant amount of heat (up to $200{ }^{\circ} \mathrm{C}$ ) on the surface of the sample as the anode producing the X-rays is typically only a few ??? from the sample. This level of heat when combined with high energy Bremsstrahlung X-rays can degrade 
the surface. Organic chemicals are therefore not routinely analyzed by non-monochromatic X-ray sources.

\section{XPS case studies}

During laser surface processing of a sample, two phenomena occurred simultaneously. The material is ablated and chemical modifications are introduced into the surface and bulk of the material. The ablation of the irradiated material depends upon input control variables such as laser wavelength, focal spot size, repetition rate, laser intensity (fluence) and the properties of the sample materials being ablated such as sensitivity and heat capacity etc. The chemical modification will be the dominating process if the laser intensity is close to the ablation threshold of the target material. If the laser intensity crosses the ablation threshold level, laser material ablation will be the dominating process resulting in change of material morphology and small chemical modifications will be observed. Ahad et. al studied the surface ablation and chemical modifications of Polytetrafluoroethylene (PTFE) samples by increasing the extreme ultraviolet (EUV) intensity [31]. The EUV source used in this study produced photons with maximum intensity around $10 \mathrm{~nm}$ wavelength, corresponding to $112 \mathrm{eV}$ photon energy. The PTFE samples were irradiated with 50, 200 and 300 EUV pulses. The changes in the surface morphology by EUV ablation were observed by SEM and AFM and the chemical modifications introduced by the EUV irradiation were examined by XPS. The SEM and AFM results demonstrated the introduction of nano- and microstructures on the EUV irradiated PTFE samples. The XPS scans were performed for binding energies extending from $0 \mathrm{eV}$ up to $700 \mathrm{eV}$ so that electrons from fluorocarbon based polymer (PTFE) can be detected. The XPS scans revealed that with increasing the intensity of the EUV beam on the PTFE samples, increase in defluorination was observed (see Table 1). This 
defluorination occurred due to breaking of carbon and fluorine bonding, consequently provide carbon-carbon bonding. The F1s emission reduced from 76 At $\%$ to 68 At $\%$ and $\mathrm{C} 1 \mathrm{~s}$ emission increased from $23 \mathrm{At} \%$ to $30 \mathrm{At} \%$. It was further observed that oxygen present in the irradiation chamber was able to incorporate into the polymer surfaces. By introducing nitrogen during EUV irradiation on PTFE samples, incorporation of nitrogen (1.1 At\%) on the polymer samples was also observed.

Table 1. Summarized XPS results for pristine and EUV irradiated PTFE polymer samples [31].

\begin{tabular}{|c|c|c|c|c|c|}
\hline Atoms & Data & Pristine & $\begin{array}{r}50 \text { EUV } \\
\text { shot }\end{array}$ & $\begin{array}{l}200 \\
\text { EUV } \\
\text { shots }\end{array}$ & $\begin{array}{l}300 \\
\text { EUV } \\
\text { shots }\end{array}$ \\
\hline \multirow{3}{*}{ F1s } & At $\%$ & 76 & 71 & 72 & 68 \\
\hline & FWHM & & 2.46 & 2.38 & 2.81 \\
\hline & Position & 689 & 688 & 688 & 689 \\
\hline \multirow{3}{*}{ O1s } & At $\%$ & & 0.51 & 0.42 & 0.64 \\
\hline & FWHM & - & 1.95 & 0.66 & 2.63 \\
\hline & Position & - & 535 & 534 & 533 \\
\hline \multirow{6}{*}{ N1s } & At $\%$ & - & - & - & 1.10 \\
\hline & FWHM & - & - & - & 0.70 \\
\hline & Position & - & - & - & 401 \\
\hline & At $\%$ & 23 & 27 & 27 & 30 \\
\hline & FWHM & 1.88 & 2.46 & 2.47 & 2.73 \\
\hline & Position & 292 & 292 & 292 & 292 \\
\hline
\end{tabular}

Nitrogen doping by EUV surface structuring was also observed in Polyvinyl fluoride (PVF) polymer by XPS surface characterisation [34]. 


\subsubsection{Secondary Ion Mass spectroscopy (SIMS)}

Secondary ion mass spectroscopy (SIMS) is a destructive analytical technique in which material is removed from a surface by ion beam sputtering and the resultant positive and negative ions are mass analysed in a mass spectrometer [101]. The technique is element-specific and is capable of detecting all elements as well as isotopes and molecular species. Of all the beam techniques it is the most sensitive with detection limits for some elements in the $10^{14}$ to $10^{15} \mathrm{~cm}^{-3}$ range if there is very little background interference signal. Lateral resolution is typically $100 \mu \mathrm{m}$ but can be as small as $0.5 \mu \mathrm{m}$ with depth resolution of 5 to $10 \mathrm{~nm}$ [50].

SIMS works by removing material from a sample by sputtering using an ion beam. The mass/charge ratio of the removed ions is analysed, detected as a mass spectrum, as a count, or displayed on a fluorescent screen [80]. Static SIMS employs very low primary ion density of around $1 \mathrm{nA} \mathrm{cm}{ }^{-2}$ and low primary ion energy $(0.5-2 \mathrm{keV})$ so that a nearly undisturbed monolayer of the surface can be analysed. Dynamic SIMS involves primary ion currents greater than $1 \mu \mathrm{A}$ $\mathrm{cm}^{-2}$ and usually more than one monolayer is removed during the analysis [72]. Dynamic SIMS can produce depth profiles, and quantitative depth profiling is unquestionably the major strength of SIMS. SIMS lends itself to investigations of grain boundary diffusion or diffusion across interfaces. It is a powerful tool for studying the transport processes in ceramics in the temperature range where diffusion distances are too small to be analysed by serial mechanical sectioning. Fielitz et al. used SIMS depth profiling to investigate oxygen grain boundary diffusion in mullite ceramics and its effect on sintering, grain growth and creep [102] .Kowalski investigated the diffusion of calcium in yttria-stabilized zirconia ceramics [103]. Haneda investigated bizmuth 
solubility and the grain boundary diffusion of oxygen ions in $\mathrm{ZnO}$ ceramics, which is important in terms of understanding electrical transport and the effects on varistor characteristics [104].

\section{SIMS case studies}

Lee et al. (2014) investigated the potential toxicity of ZincOxide nanoparticles (NPs) to human skin cells. $\mathrm{ZnO}$ nanoparticles are finding increased applications in many areas from anti-microbial coatings in food packaging, anti-bacterial coatings for surfaces, additions to waste treatment filters, additions to waste water sludge and as a sun block agent in paints and sun creams. The latter was the subject of the Lee et al (2014) study.

Time-of-flight secondary ion mass spectrometry (TOF-SIMS) was utilised as a visualisation technique to determine the cytotoxicity of the nanoparticles to the cells of choice. $\mathrm{ZnO}$ nanoparticles was synthesised from powder using a chemical reduction technique, their morphology examined using TEM. The HaCaT cells were grown to a density of $1.0 \times 10^{5}$ cell per well before addition of nanoparticles at varying concentrations of $0,5,10,50$ and $100 \mu \mathrm{g} / \mathrm{ml}$ in an aqueous solution and cultured for a further 24 hours within a $1 \times 1 \mathrm{~cm}^{2}$ silicon wafer. After culturing, the cells were then washed with PBS buffer to remove medium and additional cell residue. They were then freeze dried and subjected to modified molecular-imprint treatment, with the wafer pressed against a silicon wafer coated with a gold layer. A TOF-SIMS instrument was operated with a $\mathrm{Ga}^{+}$primary ion gun of $25 \mathrm{keV}, 1 \mathrm{pA}$ pulse current. This procedure was carried out to ensure that only the internal of the cells was imaged. An example image produced of the $\mathrm{ZnO}$ NPs acting as a tracer with HaCaT cells can be seen in figure 3.18. 


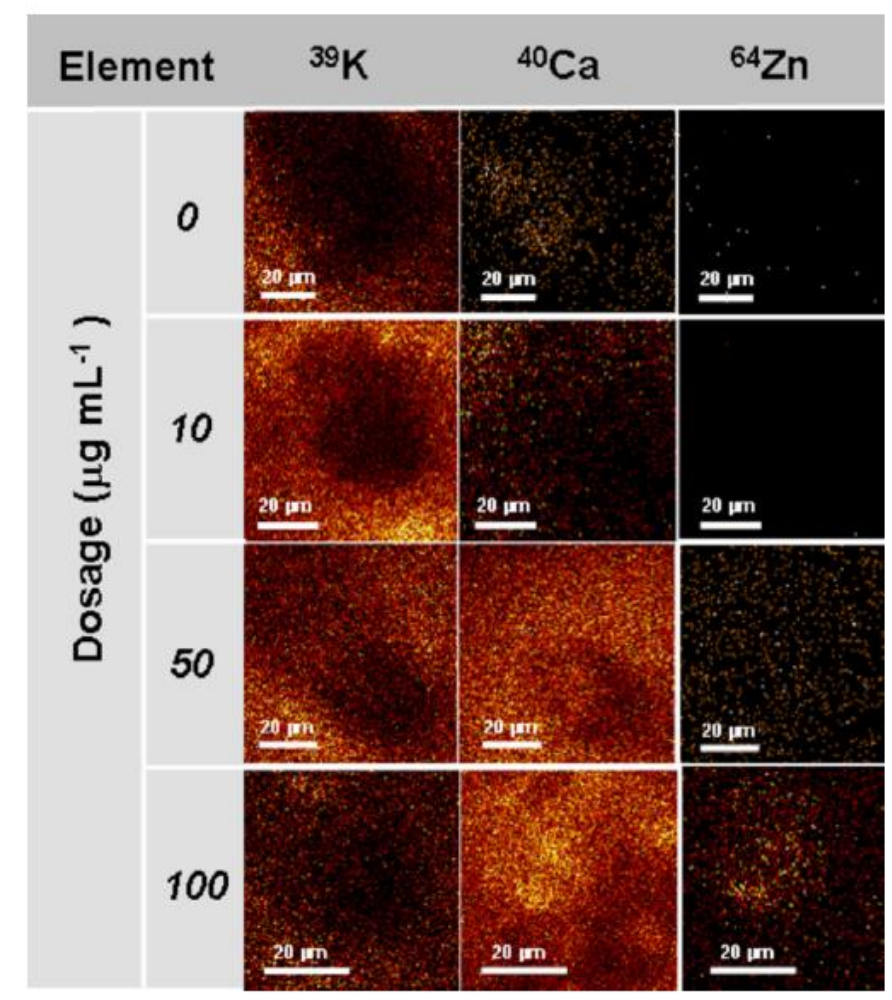

Figure 3.18 TOF-SIMS ion images of HaCaT cells treated with varying concentrations of $\mathrm{ZnO}$ NPs [105]

These images lead to conclusions that the modes of action of the NPs on the cells were membrane disruption, cytotoxic response and apoptosis. The authors concluded that TOF-SIMS was a suitable technique to offer rapid determination of cell response to NPs.

\subsubsection{Auger Electron spectroscopy (AES)}

When an electron or ion is incident on a semiconductor, it may transfer enough energy to an innershell electron to eject it from its parent atom. The atom is in an excited state, and, to lower its energy, an electron from a less tightly bound shell may fill the hole while simultaneously emitting a third electron from the atom. This ejected atom is known as an Auger electron [72]. Its energy is related specifically to the electron energy levels involved in the process and therefore, is characteristic of the atom concerned. Since the Auger process is a three-electron process, neither 
hydrogen nor helium can be detected since both have less than three electrons. AES has two distinct advantages over EDX analysis. It is a far more surface-sensitive technique. Escape depths range from less than a nanometer to a few nanometers. In EDX it can be difficult to analyse small particles on a substrate, because the electron beam passes through the particles and spreads out in the substrate below it [101]. There is the potential for chemical-state information in Auger spectroscopy, for example, the oxidation state of silicon at a $\mathrm{Si}-\mathrm{SiO}_{2}$ interface may be ascertained [72]. EDX does not provide chemical state information.

AES has found applications in measuring semiconductor composition, oxide film composition, silicides, metallization, particle analysis, and the effects of surface cleaning. AES measurements are made in a high vacuum environment $\left(10^{-12}-10^{-10}\right.$ torr $)$ to retard the formation of hydrocarbon contamination layers on the sample surface [50]. Scanning Auger Microscopy (SAM) allows surfaces to be mapped for one selected element at a time. In this mode the electron beam is scanned over a selected area. The Auger intensity is measured at each point of the area [68]. SAM requires higher beam currents and is much slower than SEM/EDX [50].

\section{AES case studies}

Rades et al. (2014) used AES to investigate the chemical structure of silica nanoparticles, identified as a high priority nanomaterial for the industrial market. Given that Auger electron spectroscopy (AES) possesses an analysis depth of only a few nanometres it is suited to examination of the chemical composition of nanoparticle surfaces. In this study particles were imaged and preselected on a TEM grid before being put forward for AES analysis, see figure 3.19. In terms of spectra the signals from $\mathrm{Si}$ and $\mathrm{O}_{2}$ are well distinguishable implying that silica has oxidized. The authors note 
that there must be a contamination layer on the bulk silica particles containing traces of iron fluorine and nitrogen.
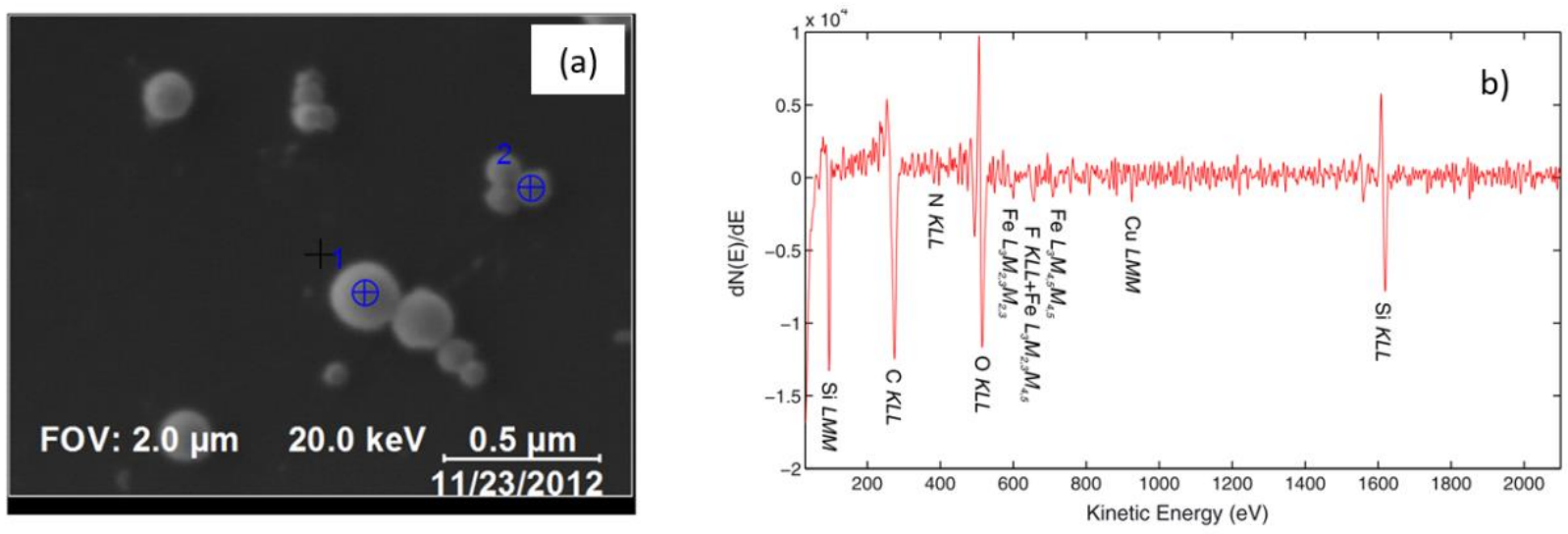

Figure 3.19 (a) Secondary electron image of the TEM grid showing the particles selected for AES analysis, and (b) the AES spectra for the selected silica particle [106] .

More recently Sharma et al. (2017) explored the synthesis of palladium (Pd) nanoparticle supported magnetic catalyst $\left(\mathrm{Pd} @ \mathrm{Fe}_{3} \mathrm{O}_{4}-\mathrm{NH}_{2} / \mathrm{Starch}\right)$. Inductively coupled plasma atomic emission spectroscopy (ICP-AES) was used to examine the Pd content that was contained in the catalyst. ICP-AES determined that $2.52 \mathrm{wt} \% \mathrm{Pd}$ was present on the catalyst. [107]. Also, Zhang et al. (2017) used ICP-AES to measure the concentration of heavy metal ions in the solution during the synthesis of $\mathrm{SnS} 2$ nanostructures.

\subsection{Conclusion}

Over the past ten years nanoparticles and nanostructures have been adopted at an ever-increasing rate throughout research and industry in many diverse fields. They have applied for improving the mechanical and electrical properties of materials as nanocomposites, and in electronics where they are being employed as printable conductive inks. They have been used in food packaging to 
improve the anti-microbial qualities of the packaging and reducing food waste. In water treatment, they have been used to coat filters and as flocculation agents. They have also been employed as paint additives to changing their optical properties. Nanoparticles and structures are being used for separation science applications, separating and detecting chemical and biological species, as well as in drug delivery via bio-tagging.

This wide adaption of nanomaterials places increasing requirements on technology to adapt and enable characterisation of the nanomaterials both in their raw and final product forms. Technology must respond to meet these demands. In this chapter, various approaches to meeting these requirements have been discussed, and with each technique it is seen that higher resolutions, larger viewing sizes and quicker scanning times are being achieved.

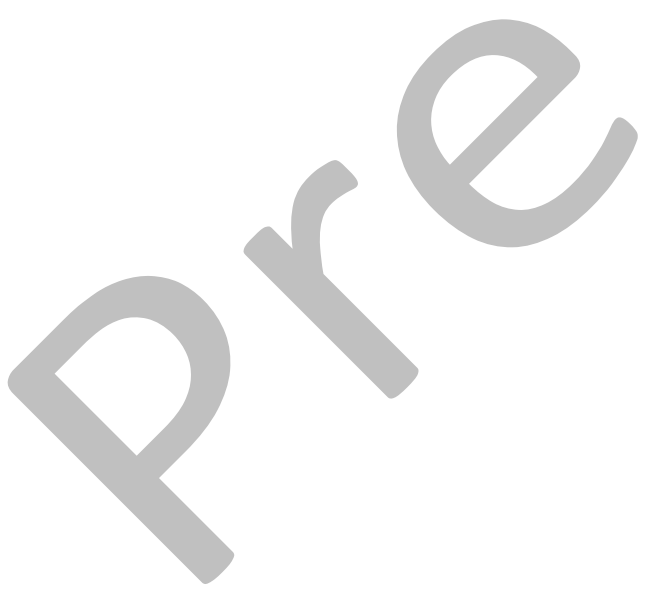




\section{References}

[1] F. Beshkar, O. Amiri, and Z. Salehi, "Synthesis of ZnSnO 3 nanostructures by using novel gelling agents and their application in degradation of textile dye," Sep. Purif. Technol., vol. 184, pp. 66-71, 2017.

[2] A. Mohammadkhani, M. Malboubi, C. Anthony, and K. Jiang, "Characterization of surface properties of ordered nanostructures using SEM stereoscopic technique," Microelectron. Eng., vol. 88, no. 8, pp. 2687-2690, 2011.

[3] E. Buhr, N. Senftleben, T. Klein, D. Bergmann, D. Gnieser, C. G. Frase, and H. Bosse, "Characterization of nanoparticles by scanning electron microscopy in transmission mode," Meas. Sci. Technol., vol. 20, no. 8, p. 84025, 2009.

[4] A. O’Shea, J. Wallace, M. Hummel, L. H. Strauss, and T. E. Kidd, "Enhanced detection of nanostructures by scanning electron microscopy using insulating materials," Micron, vol. 52-53, pp. 57-61, 2013.

[5] J. S. Villarrubia, A. E. Vlad??r, B. Ming, R. J. Kline, D. F. Sunday, J. S. Chawla, and S. List, "Scanning electron microscope measurement of width and shape of 10nm patterned lines using a JMONSEL-modeled library," Ultramicroscopy, vol. 154, pp. 15-28, 2015.

[6] Y. Jusman, S. C. Ng, and N. A. Abu Osman, "Investigation of CPD and HMDS sample preparation techniques for cervical cells in developing computer-aided screening system based on FE-SEM/EDX," Sci. World J., vol. 2014, pp. 1-11, 2014.

[7] D. D. Vaughn, O. D. Hentz, S. Chen, D. Wang, and R. E. Schaak, "Formation of SnS nanoflowers for lithium ion batteries," Chem. Commun., vol. 48, no. 45, p. 5608, 2012.

[8] J.-G. Kang, J.-G. Park, and D.-W. Kim, "Superior rate capabilities of SnS nanosheet electrodes for Li ion batteries," 2010. 
[9] Y. Wang, X. Yang, Z. Wang, X. Lv, H. Jia, J. Kong, and M. Yu, “CdS and SnS2 nanoparticles co-sensitized $\mathrm{TiO} 2$ nanotube arrays and the enhanced photocatalytic property," J. Photochem. Photobiol. A Chem., vol. 325, pp. 55-61, 2016.

[10] H. Kafashan, R. Ebrahimi-Kahrizsangi, F. Jamali-Sheini, and R. Yousefi, "Effect of Al doping on the structural and optical properties of electrodeposited SnS thin films," Phys. Status Solidi, vol. 213, no. 5, pp. 1302-1308, 2016.

[11] H. Kafashan, F. Jamali-Sheini, R. Ebrahimi-Kahrizsangi, and R. Yousefi, "Influence of growth conditions on the electrochemical synthesis of SnS thin films and their optical properties," Int. J. Miner. Metall. Mater., vol. 23, no. 3, pp. 348-357, Mar. 2016.

[12] U. Chalapathi, B. Poornaprakash, and S.-H. Park, "Chemically deposited cubic SnS thin films for solar cell applications," Sol. Energy, vol. 139, pp. 238-248, 2016.

[13] T. Sall, M. Mollar, and B. Marí, "Substrate influences on the properties of SnS thin films deposited by chemical spray pyrolysis technique for photovoltaic applications," J. Mater. Sci., vol. 51, no. 16, pp. 7607-7613, Aug. 2016.

[14] S. Li, X. Gu, Y. Zhao, Y. Qiang, S. Zhang, and M. Sui, "Enhanced visible-light photocatalytic activity and stability by incorporating a small amount of MoS2 into Ag3PO4 microcrystals," J. Mater. Sci. Mater. Electron., vol. 27, no. 1, pp. 386-392, Jan. 2016.

[15] R. V. Kashid, P. D. Joag, M. Thripuranthaka, C. S. Rout, D. J. Late, and M. A. More, "Stable Field Emission from Layered MoS2 Nanosheets in High Vacuum and Observation of 1/f Noise," Nanomater. Nanotechnol., vol. 5, p. 1, 2015.

[16] Y. M. Chen, X. Y. Yu, Z. Li, U. Paik, and X. W. (David) Lou, "Hierarchical MoS2 tubular structures internally wired by carbon nanotubes as a highly stable anode material 
for lithium-ion batteries," Sci. Adv., vol. 2, no. 7, 2016.

[17] S. Wang, X. Li, Y. Chen, X. Cai, H. Yao, W. Gao, Y. Zheng, X. An, J. Shi, and H. Chen, “A Facile One-Pot Synthesis of a Two-Dimensional MoS2/Bi2S3 Composite Theranostic Nanosystem for Multi-Modality Tumor Imaging and Therapy," Adv. Mater., vol. 27, no. 17, pp. 2775-2782, May 2015.

[18] Y. Wang, L. Yu, and X. W. D. Lou, "Synthesis of Highly Uniform MolybdenumGlycerate Spheres and Their Conversion into Hierarchical $\mathrm{MoS}_{2}$ Hollow Nanospheres for Lithium-Ion Batteries,” Angew. Chemie Int. Ed., vol. 55, no. 26, pp. 7423-7426, Jun. 2016.

[19] H. Kafashan, M. Azizieh, and H. Nasiri Vatan, "Ultrasound-assisted electrodeposition of SnS: Effect of ultrasound waves on the physical properties of nanostructured $\mathrm{SnS}$ thin films," J. Alloys Compd., vol. 686, pp. 962-968, 2016.

[20] K. K. W. Chu, J. S. Chen, L. Der Chang, and J. T. H. Tsai, "Graphene-edge probes for scanning tunneling microscopy," Optik (Stuttg)., vol. 130, pp. 976-980, 2017.

[21] A. Krupski, "Scanning tunnelling microscopy study of Au growth on Mo(110)," Surf. Sci., vol. 605, no. 3-4, pp. 424-428, 2011.

[22] A. Mehdinia, A. A. Mohammadi, S. S. H. Davarani, and M. H. Banitaba, "Application of self-assembled monolayers in the preparation of solid-phase microextraction coatings," Chromatographia, vol. 74, no. 5-6, pp. 421-427, 2011.

[23] T. F. Huerta and J. Valenzuela, "Growth of 4-aminothiophenol on iodine modified Au(100) studied by scanning tunneling microscopy," Surf. Sci., vol. 655, pp. 17-24, 2017.

[24] K. Koguchi, T. Matsumoto, and T. Kawai, "Atomic-Scale Images of the Growth Surface of Ca1-xSrxCuO2 Thin Films,” Science (80-. )., vol. 267, no. 5194, pp. 71-73, 1995. 
[25] H. S. Jung and H. J. Kim, "Initial stage of CdTe on Si(l $\left.\begin{array}{lll}1 & 0\end{array}\right)$ grown by MBE," Curr. Appl. Phys., vol. 2, no. 5, pp. 389-391, 2002.

[26] J. Seo, T.-H. Kim, and Y. Kuk, "Visualization of the inverse layer-plus-island growth in Fe islands on W(110) substrate," Curr. Appl. Phys., vol. 15, no. 9, pp. 1042-1046, 2015.

[27] Y. J. Chang and S. Phark, “Atomic-scale visualization of initial growth of perovskites on SrTiO 3 (001) using scanning tunneling microscope," Curr. Appl. Phys., vol. 17, no. 5, pp. 640-656, 2017.

[28] A. Rafati and P. Gill, "Ultrastructural characterizations of DNA nanotubes using scanning tunneling and atomic force microscopes," J. Microsc. Ultrastruct., vol. 4, no. 1, pp. 1-5, 2016.

[29] X. Chen, M. C. Davies, C. J. Roberts, S. J. B. Tendler, P. M. Williams, J. Davies, A. C. Dawkes, and J. C. Edwards, "Interpretation of tapping mode atomic force microscopy data using amplitude-phase-distance measurements," Ultramicroscopy, vol. 75, no. 3, pp. 171$181,1998$.

[30] I. Ul Ahad, A. Bartnik, H. Fiedorowicz, J. Kostecki, B. Korczyc, T. Ciach, and D. Brabazon, "Surface modification of polymers for biocompatibility via exposure to extreme ultraviolet radiation," Journal of Biomedical Materials Research - Part A, vol. 102, no. 9. pp. 3298-3310, 2014.

[31] I. U. Ahad, B. Butruk, M. Ayele, B. Budner, A. Bartnik, H. Fiedorowicz, T. Ciach, and D. Brabazon, "Extreme ultraviolet (EUV) surface modification of polytetrafluoroethylene (PTFE) for control of biocompatibility," Nucl. Instruments Methods Phys. Res. Sect. B Beam Interact. with Mater. Atoms, vol. 364, pp. 98-107, Dec. 2014.

[32] I. U. Ahad, B. Budner, B. Korczyc, H. Fiedorowicz, A. Bartnik, J. Kostecki, S. 
Burdyńska, and D. Brabazon, "Polycarbonate polymer surface modification by extreme ultraviolet (EUV) radiation,” in Acta Physica Polonica A, 2014, vol. 125, no. 4, pp. 924 928.

[33] I. Ahad, H. Fiedorowicz, B. Budner, T. J. Kaldonski, M. Vazquez, A. Bartnik, and D. Brabazon, "Extreme Ultraviolet Surface Modification of Polyethylene Terephthalate (PET) for Surface Structuring and Wettability Control,” Phys. Pol. A, vol. 129, no. 2, pp. $241-243,2016$.

[34] I. U. Ahad, B. Budner, H. Fiedorowicz, A. Bartnik, and D. Brabazon, "Nitrogen doping in biomaterials by extreme ultraviolet ( EUV ) surface modification for biocompatibility control," Eur. Cells Mater., vol. 26, no. Suppl. 6, p. 145, 2013.

[35] C. Liberatore, A. Bartnik, I. U. Ahad, M. Toufarová, I. Matulková, V. Hájková, L. Vyšín, T. Burian, L. Juha, L. Pina, A. Endo, and T. Mocek, "EUV ablation: a study of the process," in SPIE Optics + Optoelectronics, 2015, p. 951011.

[36] M. Fulwyler, Q. S. Hanley, C. Schnetter, I. T. Young, E. A. Jares-Erijman, D. J. ArndtJovin, and T. M. Jovin, "Selective photoreactions in a programmable array microscope (PAM): Photoinitiated polymerization, photodecaging, and photochromic conversion,” Cytom. Part A, vol. 67A, no. 2, pp. 68-75, Oct. 2005.

[37] M. Ilčíková, M. Danko, M. Doroshenko, A. Best, M. Mrlík, K. Csomorová, M. Šlouf, D. Chorvát, K. Koynov, and J. Mosnáček, "Visualization of carbon nanotubes dispersion in composite by using confocal laser scanning microscopy," Eur. Polym. J., vol. 79, pp. 187197, 2016.

[38] D. P. Oyarz??n, O. E. Linarez P??rez, M. L??pez Teijelo, C. Z????iga, E. Jeraldo, D. A. Geraldo, and R. Arratia-Perez, "Atomic force microscopy (AFM) and 3D confocal 
microscopy as alternative techniques for the morphological characterization of anodic TiO2 nanoporous layers," Mater. Lett., vol. 165, pp. 67-70, 2016.

[39] M. Beleites, C. Matyssek, H. H. Blaschek, and G. Seifert, "Near-field optical microscopy of femtosecond-laser-reshaped silver nanoparticles in dielectric matrix," Nanoscale Res. Lett., vol. 7, pp. 2-5, 2012.

[40] K. Bagga, R. McCann, M. Wang, a. Stalcup, M. Vázquez, and D. Brabazon, "Laser assisted synthesis of carbon nanoparticles with controlled viscosities for printing applications," J. Colloid Interface Sci., vol. 447, pp. 263-268, 2015.

[41] K. Bagga, R. McCann, F. O’Sullivan, P. Ghosh, S. Krishnamurthy, A. Stalcup, M. Vázquez, D. Brabazon, "Nanoparticle functionalized laser patterned substrate: an innovative route towards low cost biomimetic platforms," $R S C A d v$., vol. 7, no. 13, pp. 8060-8069, 2017.

[42] E. Tomaszewska, K. Soliwoda, K. Kadziola, B. Tkacz-Szczesna, G. Celichowski, M. Cichomski, W. Szmaja, and J. Grobelny, "Detection limits of DLS and UV-Vis spectroscopy in characterization of polydisperse nanoparticles colloids," J. Nanomater., vol. 2013, 2013.

[43] K. Takahashi, H. Kato, T. Saito, S. Matsuyama, and S. Kinugasa, "Precise measurement of the size of nanoparticles by dynamic light scattering with uncertainty analysis," Part. Part. Syst. Charact., vol. 25, no. 1, pp. 31-38, 2008.

[44] S. Bhattacharjee, "DLS and zeta potential - What they are and what they are not?," $J$. Control. Release, vol. 235, pp. 337-351, 2016.

[45] U. Nobbmann and A. Morfesis, "Light scattering and nanoparticles," Mater. Today, vol. 12, no. 5, pp. 52-54, 2009. 
[46] P. J. Freud, "Nanoparticle Sizing : Dynamic Light Scattering Analysis in the Frequency Spectrum Mode Provided By :," Instrumentation.

[47] S. K. Brar and M. Verma, "Measurement of nanoparticles by light-scattering techniques," TrAC - Trends Anal. Chem., vol. 30, no. 1, pp. 4-17, 2011.

[48] A. Horechyy, B. Nandan, A. Shajkumar, P. Formanek, J. Paturej, M. Stamm, and A. Fery, "In-situ monitoring of silica shell growth on PS-b-P4VP micelles as templates using DLS," Polym. (United Kingdom), vol. 107, 2016.

[49] C. R. Brundle, C. A. Evans, and S. Wilson, Encyclopedia of materials characterization : surfaces, interfaces, thin films. 1992.

[50] D. K. Schroder, Semiconductor Material and Device Characterization: Third Edition. 2005.

[51] Z. L. Wang, P. Poncharal, and W. A. De Heer, "Measuring physical and mechanical properties of individual carbon nanotubes by in situ TEM."

[52] S. K. Lim, M. Brewster, F. Qian, Y. Li, C. M. Lieber, and S. Gradečak, "Direct Correlation between Structural and Optical Properties of III-V Nitride Nanowire Heterostructures with Nanoscale Resolution," Nano Lett., vol. 9, no. 11, pp. 3940-3944, Nov. 2009.

[53] V. Barbin, "Application of cathodoluminescence microscopy to recent and past biological materials: a decade of progress," Mineral. Petrol., vol. 107, no. 3, pp. 353-362, Jun. 2013.

[54] F. Bertram, M. Müller, G. Schmidt, P. Veit, S. Petzold, S. Albert, A. M. BengoecheaEncabo, M. Á. Sánchez-Garcia, E. Calleja, and J. Christen, "Nanoscale Imaging of Structural and Optical Properties Using Helium Temperature Scanning Transmission Electron Microscopy Cathodoluminescence of Nitride Based Nanostructures," Microsc. 
Microanal., vol. 22, no. S3, pp. 600-601, 2016.

[55] E. White, A. Howkins, and C. Williams, "Investigating the Origin of Luminescence in Zinc Oxide Nanostructures With STEM-Cathodoluminescence," Microsc., 2015.

[56] G. Hanna, T. Glatzel, S. Sadewasser, N. Ott, H. P. Strunk, U. Rau, and J. H. Werner, "Texture and electronic activity of grain boundaries in $\mathrm{Cu}(\mathrm{In}, \mathrm{Ga}) \mathrm{Se} 2$ thin films," Appl. Phys. A Mater. Sci. Process., vol. 82, no. 1 SPEC. ISS., pp. 1-7, 2006.

[57] D. den Engelsen, G. R. Fern, T. G. Ireland, P. G. Harris, P. R. Hobson, A. Lipman, R. Dhillon, P. J. Marsh, and J. Silver, "Ultraviolet and blue cathodoluminescence from cubic $\mathrm{Y}_{2} \mathrm{O}_{3}$ and $\mathrm{Y}_{2} \mathrm{O}_{3}: \mathrm{Eu}^{3+}$ generated in a transmission electron microscope," J. Mater. Chem. C, vol. 4, no. 29, pp. 7026-7034, 2016.

[58] J. Wu, S. Helveg, S. Ullmann, Z. Peng, and A. T. Bell, "Growth of encapsulating carbon on supported Pt nanoparticles studied by in situ TEM," J. Catal., vol. 338, pp. 295-304, 2016.

[59] J. Wu, S. Helveg, S. Ullmann, Z. Peng, and A. Bell, "Growth of encapsulating carbon on supported Pt nanoparticles studied by in situ TEM,” J. Catal., 2016.

[60] D. Buttry, "High Resolution TEM Study on Phase Transformations in Redox Active Silver Nanoparticles," PRiME 2016/230th ECS Meet. (October 2-7, 2016), 2016.

[61] S. W. Chee, D. Loh, Z. Baraissov, P. Matsudaira, and U. Mirsaidov, "Hopping Diffusion of Gold Nanoparticles Observed with Liquid Cell TEM," Microsc. Microanal., vol. 22, no. S3, pp. 750-751, 2016.

[62] C. He, N. Zhao, C. Shi, J. Li, and H. Li, "Magnetic properties and transmission electron microscopy studies of Ni nanoparticles encapsulated in carbon nanocages and carbon nanotubes," Mater. Res. Bull., vol. 43, no. 8, pp. 2260-2265, 2008. 
[63] C. M. Wang, X. Li, Z. Wang, W. Xu, J. Liu, F. Gao, L. Kovarik, J. G. Zhang, J. Howe, D. J. Burton, Z. Liu, X. Xiao, S. Thevuthasan, and D. R. Baer, "In situ TEM investigation of congruent phase transition and structural evolution of nanostructured silicon/carbon anode for lithium ion batteries," Nano Lett., vol. 12, no. 3, pp. 1624-1632, Mar. 2012.

[64] C. Huiqun, Z. Meifang, and L. Yaogang, "Decoration of carbon nanotubes with iron oxide,” J. Solid State Chem., vol. 179, no. 4, pp. 1208-1213, Apr. 2006.

[65] J.-P. Salvetat, J.-M. Bonard, N. H. Thomson, A. J. Kulik, L. Forró, W. Benoit, and L. Zuppiroli, "Mechanical properties of carbon nanotubes," Appl. Phys. A Mater. Sci. Process., vol. 69, no. 3, pp. 255-260, Sep. 1999.

[66] M. V. Jose, B. W. Steinert, V. Thomas, D. R. Dean, M. A. Abdalla, G. Price, and G. M. Janowski, "Morphology and mechanical properties of Nylon 6/MWNT nanofibers," Polymer (Guildf)., vol. 48, no. 4, pp. 1096-1104, 2007.

[67] C. R. Musil, J. L. Bartelt, and J. Melngailis, "Focused ion beam microsurgery for electronics," IEEE Electron Device Lett., vol. 7, no. 5, pp. 285-287, May 1986.

[68] C. Boit, "New physical techniques for IC functional analysis of on-chip devices and interconnects," Appl. Surf. Sci., vol. 252, no. 1, pp. 18-23, 2005.

[69] R. Schlangen, U. Kerst, C. Boit, T. Malik, R. Jain, and T. Lundquist, Microelectronics and reliability., vol. 47. Pergamon Press, 1964.

[70] A. V Stanishevsky, "Focused Ion Beam Nanofabrication," vol. 3, no. March, 2016.

[71] R. Wierzbicki, C. Købler, M. Jensen, and J. Łopacińska, "Mapping the complex morphology of cell interactions with nanowire substrates using FIB-SEM,” PLoS, 2013.

[72] J. TE., Semiconductor science; growth and characterisation techniques. Harlow, Essex, UK: Prentice Hall, 1998. 
[73] J. Liu, U. Khan, J. Coleman, B. Fernandez, P. Rodriguez, S. Naher, and D. Brabazon, "Graphene oxide and graphene nanosheet reinforced aluminium matrix composites: Powder synthesis and prepared composite characteristics," Mater. Des., vol. 94, pp. 8794, 2016.

[74] Z. Wang, J. Shu, Q. Zhu, B. Cao, and H. Chen, "Graphene-nanosheet-wrapped LiV 3 O 8 nanocomposites as high performance cathode materials for rechargeable lithium-ion batteries," J. Power, 2016.

[75] X. Lu and H. Dou, "Simple and mass-produced mechanochemical preparation of graphene nanosheet/polyaniline composite assisted with bifunctional ionic liquid," Funct. Mater. Lett., 2016.

[76] X. Feng, W. Xing, L. Song, Y. Hu, and K. Liew, "TiO 2 loaded on graphene nanosheet as reinforcer and its effect on the thermal behaviors of poly (vinyl chloride) composites," Chem. Eng. J., 2015.

[77] B. Qiu, Y. Zhou, Y. Ma, X. Yang, W. Sheng, and M. Xing, "Facile synthesis of the Ti3+ self-doped TiO2-graphene nanosheet composites with enhanced photocatalysis," Sci. Rep., 2015.

[78] S. Fu, N. Li, K. Wang, Q. Zhang, and Q. Fu, "Reduction of graphene oxide with the presence of polypropylene micro-latex for facile preparation of polypropylene/graphene nanosheet composites," Colloid Polym. Sci., 2015.

[79] R. Haul, “S. J. Gregg, K. S. W. Sing: Adsorption, Surface Area and Porosity. 2. Auflage, Academic Press, London 1982. 303 Seiten, Preis: \$ 49.50,” Berichte der Bunsengesellschaft für Phys. Chemie, vol. 86, no. 10, pp. 957-957, Oct. 1982.

[80] P. (Paul A. . Webb, C. Orr, and Micromeritics Instrument Corporation., Analytical 
methods in fine particle technology. Micromeritics Instrument Corp, 1997.

[81] M. Terock, C. H. Konrad, R. Popp, R. Völkl, F. Yang, D. W. McComb, M. J. Mills, and U. Glatzel, "Tailored platinum-nickel nanostructures on zirconia developed by metal casting, internal oxidation and dealloying," Corros. Sci., vol. 112, pp. 246-254, 2016.

[82] M. M. Seraji, N. S. Ghafoorian, and A. R. Bahramian, "Investigation of microstructure and mechanical properties of novolac/silica and $\mathrm{C} / \mathrm{SiO} 2 / \mathrm{SiC}$ aerogels using mercury porosimetry method," J. Non. Cryst. Solids, vol. 435, pp. 1-7, 2016.

[83] J. Goldstein, Scanning electron microscopy and $x$-ray microanalysis. Kluwer Academic/Plenum Publishers, 2003.

[84] E. Lifshin and Wiley InterScience (Online service), X-ray characterization of materials. Wiley-VCH, 1999.

[85] M. A. Khan, S. Member, and R. Zheng, "Nanostructural Analysis of CMOS-MEMSBased Digital Microphone for Performance Optimization,” vol. 15, no. 6, pp. 849-855, 2016.

[86] M. Dastkhoon, M. Ghaedi, A. Asfaram, and A. Goudarzi, "Improved adsorption performance of nanostructured composite by ultrasonic wave: Optimization through response surface methodology, Isotherm and kinetic studies," Ultrasonics, 2016.

[87] R. Balow, E. Tomlinson, and M. Abu-Omar, "Solution-based synthesis and characterization of earth abundant $\mathrm{Cu} 3$ (As, Sb) Se 4 nanocrystal alloys: towards scalable room-temperature thermoelectric devices," J. Mater., 2016.

[88] G. Di Girolamo, A. Brentari, and E. Serra, "Some recent findings on the use of SEM-EDS in microstructural characterisation of as-sprayed and thermally aged porous coatings: a short review," 2016. 
[89] K. Edalati, H. Emami, Y. Ikeda, H. Iwaoka, and I. Tanaka, "New nanostructured phases with reversible hydrogen storage capability in immiscible magnesium-zirconium system produced by high-pressure torsion," Acta Mater., 2016.

[90] A. Dong, X. Ye, H. Li, Y. Zhang, and G. Wang, "Micro/nanostructured hydroxyapatite structurally enhances the immobilization for $\mathrm{Cu}$ and $\mathrm{Cd}$ in contaminated soil," J. Soils Sediments, 2016.

[91] Y. Liu, G. Zhu, J. Gao, M. Hojamberdiev, and R. Zhu, "Enhanced photocatalytic activity of Bi 4 Ti 3 O 12 nanosheets by Fe 3+-doping and the addition of Au nanoparticles: Photodegradation of Phenol and bisphenol A," Appl. Catal. B, 2017.

[92] X. Fan, Y. Cui, P. Liu, L. Gou, L. Xu, and D. Li, "Electrochemical construction of threedimensional porous Mn 3 O 4 nanosheet arrays as an anode for the lithium ion battery," Phys. Chem. Chem., 2016.

[93] K. Sasaki, K. Matsubara, S. Kawamura, and K. Saito, "Synthesis of copper nanoparticles within the interlayer space of titania nanosheet transparent films," J. Mater., 2016.

[94] S. Kim, S. Yook, A. Kannan, S. Kim, and C. Park, "Enhancement of the electrochemical performance of silicon anodes through alloying with inert metals and encapsulation by graphene nanosheets," Electrochimica, 2016.

[95] O. Mehraj, B. Pirzada, N. Mir, M. Khan, and S. Sabir, “A highly efficient visible-lightdriven novel pn junction Fe 2 O 3/BiOI photocatalyst: Surface decoration of BiOI nanosheets with Fe 2 O 3 nanoparticles," Appl. Surf. Sci., 2016.

[96] T. Pardo, D. Martínez-Fernández, and C. de la Fuente, "Maghemite nanoparticles and ferrous sulfate for the stimulation of iron plaque formation and arsenic immobilization in Phragmites australis,” Environmental, 2016. 
[97] J. Landers, J. Colon-Ortiz, and K. Zong, "In Situ Growth and Characterization of Metal Oxide Nanoparticles within Polyelectrolyte Membranes,” Angew. Chemie, 2016.

[98] V. Souza, J. Scholten, and D. Weibel, "Hybrid tantalum oxide nanoparticles from the hydrolysis of imidazolium tantalate ionic liquids: efficient catalysts for hydrogen generation from ethanol/water solutions," J. Mater., 2016.

[99] X. Li, Z. Niu, J. Jiang, and L. Ai, "Cobalt nanoparticles embedded in porous N-rich carbon as an efficient bifunctional electrocatalyst for water splitting," J. Mater. Chem. A, 2016.

[100] J. Matthew, "Surface analysis by Auger and x-ray photoelectron spectroscopy. D. Briggs and J. T. Grant (eds). IMPublications, Chichester, UK and SurfaceSpectra, Manchester, UK, 2003. 900 pp., ISBN 1-901019-04-7, 900 pp,’ Surf. Interface Anal., vol. 36, no. 13, pp. 1647-1647, 2004.

[101] X.-L. Gao, J.-S. Pan, and C.-Y. Hsu, “Laser-Fluoride Effect on Root Demineralization,” J. Dent. Res., vol. 85, no. 10, pp. 919-923, Oct. 2006.

[102] V. Balter and B. Reynard, "Secondary ionization mass spectrometry imaging of dilute stable strontium labeling in dentin and enamel," Bone, vol. 42, no. 1, pp. 229-234, Jan. 2008.

[103] W. Qian, M. Murakami, Y. Ichikawa, and Y. Che, "Highly efficient and controllable PEGylation of gold nanoparticles prepared by femtosecond laser ablation in water," $J$. Phys. Chem. C, vol. 115, no. 47, pp. 23293-23298, 2011.

[104] J. F. Watts and J. Wolstenholme, An introduction to surface analysis by XPS and AES. J. Wiley, 2003.

[105] P. L. Lee, B. C. Chen, G. Gollavelli, S. Y. Shen, Y. S. Yin, S. L. Lei, C. L. Jhang, W. R. 
Lee, and Y. C. Ling, "Development and validation of TOF-SIMS and CLSM imaging method for cytotoxicity study of $\mathrm{ZnO}$ nanoparticles in HaCaT cells," J. Hazard. Mater., vol. 277, pp. 3-12, 2014.

[106] S. Rades, T. Wirth, and W. Unger, "Investigation of silica nanoparticles by Auger electron spectroscopy (AES),” Surf. Interface Anal., vol. 46, no. 10-11, pp. 952-956, 2014.

[107] H. Sharma, M. Bhardwaj, M. Kour, and S. Paul, "Highly efficient magnetic Pd ( 0 ) nanoparticles stabilized by amine functionalized starch for organic transformations under mild conditions," Mol. Catal., vol. 435, pp. 58-68, 2017.

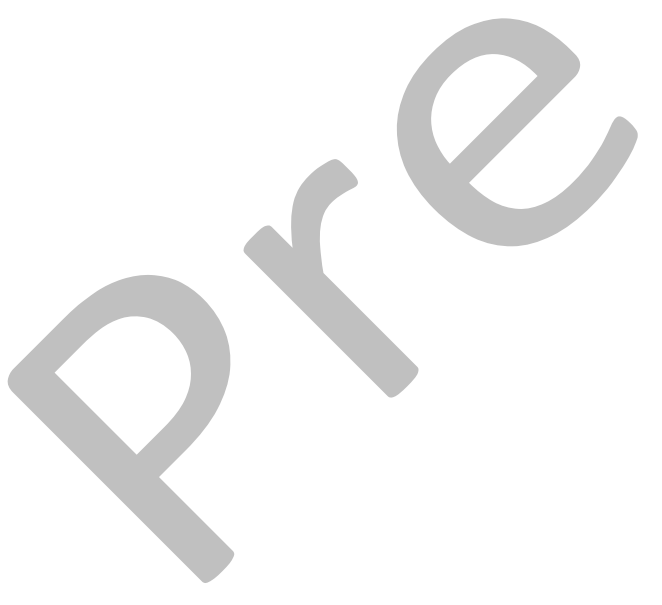

